\title{
Dynamic Average Consensus under Limited Control Authority and Privacy Requirements*
}

\author{
Solmaz S. Kia, Jorge Cortés, Sonia Martínez \\ Department of Mechanical and Aerospace Engineering, \\ University of California San Diego, La Jolla, CA 92093, USA
}

\begin{abstract}
This paper introduces a novel continuous-time dynamic average consensus algorithm for networks whose interaction is described by a strongly connected and weight-balanced directed graph. The proposed distributed algorithm allows agents to track the average of their dynamic inputs with some steady-state error whose size can be controlled using a design parameter. This steady-state error vanishes for special classes of input signals. We analyze the asymptotic correctness of the algorithm under time-varying interaction topologies and characterize the requirements on the stepsize for discrete-time implementations. We show that our algorithm naturally preserves the privacy of the local input of each agent. Building on this analysis, we synthesize an extension of the algorithm that allows individual agents to control their own rate of convergence towards agreement and handle saturation bounds on the driving command. Finally, we show that the proposed extension additionally preserves the privacy of the transient response of the agreement states and the final agreement value from internal and external adversaries. Numerical examples illustrate the results.
\end{abstract}

Keywords: dynamic average consensus; time-varying input signals; directed graphs; rate of con-

*under review in International Journal of Robust and Nonlinear Control 
vergence; limited control authority; privacy preservation

\section{Introduction}

This paper studies the dynamic average consensus problem for a network of autonomous agents. Given a set of time-varying signals, one per agent, this problem consists of designing a distributed algorithm that allow agents to track the time-varying average of the signals using only information from neighbors. Solutions to this problem are of interest in scenarios that require the fusion of dynamic and evolving information collected by multiple agents. Examples include multi-robot coordination [1], distributed spatial estimation [2, 3], sensor fusion [4, 5], feature-based map merging [6], and distributed tracking [7]. We are particularly interested in algorithmic solutions that allow agents to adjust the rate of convergence towards agreement, are able to handle constraints on actuation, and preserve the privacy of the information available to them against adversaries.

\section{Literature review.}

Consensus problems have been intensively studied over the last years. The main body of work focuses on the static case, where agents aim to reach consensus on a function depending on initial static values, see e.g. $[8,9,10,11,12]$ and references therein. In contrast, the literature on dynamic consensus is not as rich. The initial work [13] proposes a dynamic average consensus algorithm that under proper initialization is able to track, with zero steady-state error, the average of dynamic inputs whose Laplace transfer functions have at most one pole at the origin and the rest of the poles are in the left half-plane. In [4], the authors generalize the static consensus algorithm of [14] to track the average of inputs with bounded derivatives which differ by a zero-mean Gaussian noise. The algorithm acts as a low-pass filter that allows agents to track the average of dynamic inputs with a non-zero steady-state error, which vanishes in the absence of noise. Using input-to-state stability analysis, [15] proposes a proportional-integral algorithm to solve the dynamic consensus problem which, from any initial condition, converges with non-zero steady-state error if the signals are slowly 
time-varying, and exactly if the signals are static. This algorithm is generalized in [16] to achieve zero-error dynamic average consensus of a special class of time-varying input signals whose Laplace transform is a rational function with no poles in the left-hand complex plane. The proposed algorithm employs frequency-domain tools and exploits the properties of the inputs' Laplace transforms. All the algorithms mentioned above are designed in continuous time and work for networks with a fixed, connected, and undirected graph topology. The results of [15] can be applied to networks with a strongly connected and weight-balanced digraph topology provided each agent can communicate with its out-neighbors and knows the weights of its incoming edges. Such requirement may be hard to satisfy in scenarios where the topology is changing. The work [17] develops an alternative class of discrete-time dynamic average consensus algorithms whose convergence analysis relies on inputto-output stability properties in the presence of external disturbances. With a proper initialization of the states, the proposed schemes can track, with a bounded steady-state error, the average of the time-varying inputs whose $n$ th-order difference is bounded. If the $n$ th-order difference is asymptotically zero, the estimates of the average converge to the true average asymptotically with one timestep delay. Other classes of algorithms related to our work are leader-follower algorithms for networks of mobile agents with integrator dynamics, e.g., see [18, 19], and robust average consensus algorithms in the presence of additive input disturbances [20]. In the former scenario, agents reach consensus by following the input signal of the leader agent(s), instead of converging to the average of input signals across the network. In the latter case, the algorithm performance achieving consensus is analyzed in the presence of dynamic external disturbances. A common limitation of the works cited above is the lack of consideration of restrictions on the rate of convergence of individual agents, bounded control authority, or privacy issues. Regarding the latter, the above algorithms require agents to share their agreement state with their neighbors, and, in some cases, even their local inputs. Therefore, if adversaries are able to listen to the exchanged messages, they could infer local inputs, sensitive transient responses and final agreement states of the network. 


\section{Statement of contributions.}

We begin by providing a formal statement of the dynamic average consensus problem for a multiagent system, paying special attention to the rate of convergence, limits on control actuation, and the preservation of privacy. Our starting point is the introduction of a continuous-time algorithm that allows the group of agents communicating over a strongly connected and weight-balanced digraph to track the average of their reference inputs with some steady-state error. We carefully characterize the asymptotic convergence properties of the proposed strategy, including its rate of convergence, its robustness against initialization errors, and its amenability to discrete-time implementations. We also discuss how the algorithm performance (specifically, the steady-state error and the transient response) can be tuned via two design parameters. For special classes of inputs, which include static inputs and dynamic inputs which differ by a constant value, we show that the steady-state error vanishes. We also establish the algorithm correctness under time-varying network topologies that remain weight-balanced and are infinitely often jointly strongly connected. Our next step is the introduction of an extension of the proposed dynamic average consensus algorithm to include a local first-order filter at each agent. We show how this extension allows individual agents to tune their rate of convergence towards agreement without affecting the rest of the network or changing the ultimate tracking error bound. We also establish that, under limited control authority, this extension has the same correctness guarantees as the original algorithm as long as the input signals are bounded with a bounded relative growth. Several simulations illustrate our results. Our final step is the characterization of the privacy-preservation properties of the proposed dynamic average consensus algorithms. We consider adversaries who aim to retrieve information about the inputs, their average, or the state trajectories. These adversaries might be inside (internal) or outside (external) the network, do not interfere with the algorithm execution, and may have access to different levels of information, such as knowledge of certain parts of the graph topology, the algorithm design parameters, initial conditions, or the history of communication messages. We show how the 
proposed algorithms naturally preserve the privacy of the input of each agent against any adversary. Moreover, we establish that the extension that incorporates local first-order filters protects the privacy of the agreement state trajectories against any adversary by adding a common signal to the messages transmitted among neighbors. This strategy also preserves the privacy of the final agreement value against external adversaries.

\section{Organization.}

Section 2 introduces basic notation, graph-theoretic concepts, and the model of time-varying networks. Section 3 formally introduces the dynamic consensus problems of interest. Section 4 presents our dynamic average consensus algorithm, establishes its correctness, and analyzes its properties regarding changing interaction topologies, discrete-time implementations, and rate of convergence. Section 5 introduces a modified version which enables agents to opt for a slower rate of convergence and solves the consensus problem in the presence of bounded control commands. Section 6 considers the privacy preservation properties of the proposed algorithms. Section 7 presents simulations illustrating our results. Finally, Section 8 gathers our conclusions and ideas for future work.

\section{Preliminaries}

In this section, we introduce basic notation, concepts from graph theory used throughout the paper, and our model for networks with time-varying interaction topologies.

\subsection{Notational conventions}

The vector $\mathbf{1}_{n}$ is the vector of $n$ ones, $\mathbf{0}_{n}$ is the vector of $n$ zeros, and $\boldsymbol{I}_{n}$ is the identity matrix with dimension $n \times n$. We denote by $\boldsymbol{A}^{\top}$ the transpose of matrix $\boldsymbol{A}$. For a square matrix $\boldsymbol{A}$ we define $\operatorname{Sym}(\boldsymbol{A})=\frac{1}{2}\left(\boldsymbol{A}+\boldsymbol{A}^{\top}\right)$. We use $\operatorname{Diag}\left(\boldsymbol{A}_{1}, \cdots, \boldsymbol{A}_{N}\right)$ to represent the block-diagonal matrix constructed from matrices $\boldsymbol{A}_{1}, \ldots, \boldsymbol{A}_{N}$. We define $\boldsymbol{\Pi}_{n}=\boldsymbol{I}_{n}-\frac{1}{n} \mathbf{1}_{n} \mathbf{1}_{n}^{\top}$. We denote the induced two-norm of a real matrix $\boldsymbol{A}$ by $\|\boldsymbol{A}\|$, i.e., $\|\boldsymbol{A}\|=\sigma_{\max }(\boldsymbol{A})$, where $\sigma_{\max }$ is the maximum singular 
value of $\boldsymbol{A}$. The spectral radius of a square matrix $\boldsymbol{A}$ is represented by $\rho(\boldsymbol{A})$. For a vector $\boldsymbol{u}$, we use $\|\boldsymbol{u}\|$ to denote the standard Euclidean norm, i.e., $\|\boldsymbol{u}\|=\sqrt{\boldsymbol{u}^{\top} \boldsymbol{u}}$. For vectors $\boldsymbol{u}_{1}, \cdots, \boldsymbol{u}_{N}$, we let $\left(\boldsymbol{u}_{1}, \cdots, \boldsymbol{u}_{N}\right)$ represent their aggregated vector. For a complex variable $c, \Re(c)$ indicates its real part. For a scalar variable $u$, the saturation function with limit $0<\bar{u}<\infty$ is indicated by $\operatorname{sat}_{\bar{u}}(u)$, i.e., $\operatorname{sat}_{\bar{u}}(u)=\operatorname{sign}(u) \min \{|u|, \bar{u}\}$. We let $\delta_{1}(\epsilon) \in O\left(\delta_{2}(\epsilon)\right)$ denote the fact that there exist positive constants $c$ and $k$ such that $\left|\delta_{1}(\epsilon)\right| \leq k\left|\delta_{2}(\epsilon)\right|, \forall|\epsilon|<c$. For network-related variables, the local variables of each agent are distinguished by a superscript, e.g., $u^{i}(t)$ is the local dynamic input of agent $i$. If $p^{i} \in \mathbb{R}$ is a local variable at agent $i$, the aggregated $p^{i}$ 's are represented by $\boldsymbol{p}=\left(p^{1}, \ldots, p^{N}\right) \in \mathbb{R}^{N}$. Our analysis involves linear systems of the form

$$
\dot{\boldsymbol{x}}(t)=\boldsymbol{A x}(t)+\boldsymbol{B} \boldsymbol{u}(t),
$$

where states $\boldsymbol{x}(t)$ take values in the Euclidean space $\mathbb{R}^{n}$, and inputs are measurable locally essentially bounded maps $\boldsymbol{u}:[0, \infty) \rightarrow \mathbb{R}^{m}$. The zero-system associated to (1) is by definition the system with no inputs, i.e., $\dot{\boldsymbol{x}}=\boldsymbol{A} \boldsymbol{x}$. We denote by $\|\boldsymbol{u}\|_{\text {ess }}$, the (essential) supremum norm, i.e., $\|\boldsymbol{u}\|_{\text {ess }}=$ $\sup \{\|\boldsymbol{u}(t)\|, t \geq 0\}<\infty$. The convergence rate of a stable linear system $\dot{\boldsymbol{x}}=\boldsymbol{A} \boldsymbol{x}$ is

$$
r=\inf \left\{\chi>0 \mid \exists \kappa>0 \text { such that }\|\boldsymbol{x}(t)\| \leq \kappa\|\boldsymbol{x}(0)\| \mathrm{e}^{-\chi t}, \quad t \geq 0\right\}
$$

Here, $\boldsymbol{x}(t)$ is the solution of the system when it starts from any initial state $\boldsymbol{x}(0) \in \mathbb{R}^{n}$. This definition implies that for a linear time-invariant dynamical system, the rate of convergence is the least negative real part of the eigenvalues of the system matrix.

\subsection{Graph theory}

We briefly review some basic concepts from the graph, see e.g. [12]. A directed graph, or simply a digraph, is a pair $\mathcal{G}=(\mathcal{V}, \mathcal{E})$, where $\mathcal{V}=\{1, \ldots, N\}$ is the node set and $\mathcal{E} \subseteq \mathcal{V} \times \mathcal{V}$ is the edge set. An edge from $i$ to $j$, denoted by $(i, j)$, means that agent $j$ can send information to agent $i$. For an edge $(i, j) \in \mathcal{E}, i$ is called an in-neighbor of $j$, and $j$ is called an out-neighbor of $i$. A digraph $\mathcal{G}^{\prime}=\left(\mathcal{V}, \mathcal{E}^{\prime}\right)$ is a spanning subgraph of a digraph $\mathcal{G}=(\mathcal{V}, \mathcal{E})$ if $\mathcal{E}^{\prime} \subset \mathcal{E}$. A graph is undirected if 
$(i, j) \in \mathcal{E}$ anytime $(j, i) \in \mathcal{E}$. Given digraphs $\mathcal{G}_{i}=\left(\mathcal{V}, \mathcal{E}_{i}\right), i \in\{1, \ldots, m\}$, defined on same node set, the joint digraph of these digraphs is the union $\cup_{i=1}^{n} \mathcal{G}_{i}=\left(\mathcal{V}, \mathcal{E}_{1} \cup \mathcal{E}_{2} \cup \cdots \cup \mathcal{E}_{m}\right)$. A directed path is an ordered sequence of vertices such that any ordered pair of vertices appearing consecutively is an edge of the digraph. A directed tree is an acyclic digraph with the following property: there exists a node, called the root, such that any other node of the digraph can be reached by one and only one directed path starting at the root. A directed spanning tree of a digraph is a spanning subgraph that is a directed tree. A digraph is called strongly connected if for every pair of vertices there is a directed path between them.

A weighted digraph is a triplet $\mathcal{G}=(\mathcal{V}, \mathcal{E}, \mathbf{A})$, where $(\mathcal{V}, \mathcal{E})$ is a digraph and $\mathbf{A} \in \mathbb{R}^{N \times N}$ is a weighted adjacency matrix with the property that $a_{i j}>0$ if $(i, j) \in \mathcal{E}$ and $a_{i j}=0$, otherwise. We use $\Gamma(\mathbf{A})$ to denote a digraph induced by a given adjacency matrix $\mathbf{A}$. A weighted digraph is undirected if $a_{i j}=a_{j i}$ for all $i, j \in \mathcal{V}$. The weighted out-degree and weighted in-degree of a node $i$, are respectively, $\mathrm{d}^{\text {in }}(i)=\sum_{j=1}^{N} a_{j i}$ and $\mathrm{d}^{\text {out }}(i)=\sum_{j=1}^{N} a_{i j}$. We let $\mathrm{d}_{\max }^{\text {out }}=\max _{i \in\{1, \ldots, N\}} \mathrm{d}^{\text {out }}(i)$ denote the maximum weighted out-degree. A digraph is weight-balanced if at each node $i \in \mathcal{V}$, the weighted out-degree and weighted in-degree coincide (although they might be different across different nodes). The outdegree matrix $\mathbf{D}^{\text {out }}$ is the diagonal matrix with entries $\mathbf{D}_{i i}^{\text {out }}=\mathrm{d}^{\text {out }}(i)$, for all $i \in \mathcal{V}$. The (out-) Laplacian matrix is $\mathbf{L}=\mathbf{D}^{\text {out }}-\mathbf{A}$. Note that $\mathbf{L} \mathbf{1}_{N}=0$. A weighted digraph $\mathcal{G}$ is weight-balanced if and only if $\mathbf{1}_{N}^{T} \mathbf{L}=0$. Based on the structure of $\mathbf{L}$, at least one of the eigenvalues of $\mathbf{L}$ is zero and the rest of them have nonnegative real parts. We denote the eigenvalues of $\mathbf{L}$ by $\lambda_{i}, i \in\{1, \ldots, N\}$, where $\lambda_{1}=0$ and $\Re\left(\lambda_{i}\right) \leq \Re\left(\lambda_{j}\right)$, for $i<j$. For a strongly connected digraph, zero is a simple eigenvalue of $\mathbf{L}$. We denote the eigenvalues of $\operatorname{Sym}(\mathbf{L})$ by $\hat{\lambda}_{i}, i \in\{1, \ldots, N\}$. For a strongly connected and weight-balanced digraph, zero is a simple eigenvalue of $\operatorname{Sym}(\mathbf{L})$. For such a digraph, we order the eigenvalues of $\operatorname{Sym}(\mathbf{L})$ as $\hat{\lambda}_{1}=0<\hat{\lambda}_{2} \leq \hat{\lambda}_{3} \leq \cdots \leq \hat{\lambda}_{N}$. 


\subsection{Time-varying interactions via switched systems}

Here, we introduce our model of networks with fixed number of agents but time-varying interaction topologies. Let $(\mathcal{V}, \mathcal{E}(t), \mathbf{A}(t))$ be a time-varying digraph, where the nonzero entries of the adjacency matrix are uniformly lower and upper bounded, i.e., $a_{i j}(t) \in[\underline{a}, \bar{a}]$, where $0<\underline{a} \leq \bar{a}$, if $(j, i) \in \mathcal{E}(t)$, and $a_{i j}=0$ otherwise. Our model of time-varying networks is then $\mathcal{G}(t)=\Gamma\left(\mathbf{A}_{\sigma(t)}\right), t \geq 0$, with $\sigma:[0, \infty) \rightarrow \mathcal{P}=\{1, \ldots, m\}$ a piecewise constant signal belonging to some switching set $\mathcal{S}$. Here, $m$ can be infinity. In our developments later, we provide precise specifications for $\mathcal{S}$. By piecewise constant, we mean a signal that only has a finite number of discontinuities in any finite time interval and that is constant between consecutive discontinuities (no chattering). Without loss of generality, we assume that switching signals are continuous from the right. The uniform stability of switched linear systems with time-dependent switching signals (where uniformity refers to the multiple solutions that can be obtained as the switching signal ranges over a switching set) is characterized by the following result.

Lemma 2.1 (Asymptotic stability of switched linear systems implies exponential stability [21]): For linear switched systems with trajectory-independent switching, uniform asymptotic stability is equivalent to exponential stability.

We end this section by introducing the following notations. Given a time-varying digraph, we denote by $\cup_{t_{1}}^{t_{2}} \mathcal{G}(t)$ the joint digraph in the time interval $\left[t_{1}, t_{2}\right)$ where $t_{1}<t_{2}<+\infty$. We say a time-varying graph $\mathcal{G}(t)$ is jointly strongly connected over the time-interval $\left[t_{1}, t_{2}\right)$ if $\cup_{t_{1}}^{t_{2}} \mathcal{G}(t)$ is strongly connected. The time instants at which the switching signal $\sigma$ is discontinuous are called switching times and are denoted by $t_{0}, t_{1}, t_{2}, \cdots$, where $t_{0}=0$. We use $\mathbf{L}_{\sigma}$ to represent the out-Laplacian of the digraph $\Gamma\left(\mathbf{A}_{\sigma}\right)$. 


\section{Problem statement}

We consider a network of $N$ agents with single-integrator dynamics given by

$$
\dot{x}^{i}=c^{i}, \quad i \in\{1, \ldots, N\},
$$

where $x^{i} \in \mathbb{R}$ is the agreement state and $c^{i} \in \mathbb{R}$ is the driving command of agent $i$. The network interaction topology is modeled by a weighted digraph $\mathcal{G}$. Agent $i \in\{1, \ldots, N\}$ has access to a time-varying input signal $u^{i}:[0, \infty) \rightarrow \mathbb{R}$. The problem we are interested in solving is the following.

Problem 1 (Dynamic average consensus): Let $\mathcal{G}$ be strongly connected and weight-balanced. Design a distributed algorithm such that each agent's state $x^{i}(t)$ asymptotically tracks the average $\frac{1}{N} \sum_{j=1}^{N} u^{j}(t)$ of the inputs.

This problem finds numerous applications in networks of multiple agents that have access to partial and evolving information, and aim to combine it in a dynamic fashion. Examples are numerous and include data fusion, spatial estimation, and localization and mapping, to name a few. The algorithm design amounts to specifying a suitable driving command $c^{i}$ for each agent $i \in\{1, \ldots, N\}$. By distributed, we mean that agent $i$ only interacts with its out-neighbors. In addition, we also consider variations of the problem above that are intended to satisfy some practical issues that arise in using the consensus algorithm in applications where the agent state corresponds to a physical quantity such as position or velocity in motion coordination of autonomous mobile agents. In such applications, a genuine concern is whether the command $c^{i}$ dictated by the consensus algorithm can be implemented given the physical limitation of the actuation systems. This motivates us to formulate the following variation of Problem 1.

Problem 2 (Dynamic average consensus with controllable rate of convergence): Solve Problem 1 such that each agent converges at its own desired rate of convergence.

By giving a freedom to choose their desired rate of convergence, we allow agents with limited control authority to opt for a slow rate of convergence. We can also use the control over the individual rate 
of convergence of agents in scheduling different time of arrivals. This can benefit applications such as payload delivery or aerial surveillance. Although reducing the rate of convergence helps with cases where control authority is limited, there is no guarantee that control bounds, if present, would be satisfied. This motivates us to formulate the next problem.

Problem 3 (Dynamic average consensus with limited control authority): Solve Problem 1 under bounded driving commands, i.e., $\dot{x}^{i}=\operatorname{sat}_{\bar{c}^{i}}\left(c^{i}\right)$ for all $i \in\{1, \ldots, N\}$.

Finally, we consider the problem of dynamic average consensus with privacy preservation in the presence of adversaries. Our motivation to study such properties stems from the fact that privacy guarantees on a distributed algorithm facilitate the agent participation in the completion of cooperative tasks. In an average consensus problem, the privacy concern of agents can be local (e.g., some or all of the agents do not want to reveal their local inputs to the outside world) or global (e.g., all agents do not want to reveal their agreement value to agents outside network). We consider adversaries inside or outside the network that do not interfere with the algorithm implementation but seek to steal information about the inputs, agreement value, or the agreement state trajectories of the individual agents. The information these adversaries can access includes the time history of intra network communication messages, partial or full knowledge about the communication topology, and the algorithm design parameters, and/or its initial conditions.

Problem 4 (Dynamic average consensus with privacy preservation): Solve Problems 1-3 such that the following privacy requirements are satisfied

(a) the local inputs of the agents should not be revealed or be reconstructible by any adversary;

(b) the agreement value should not be revealed to or be reconstructible by external adversaries;

(c) the agreement state should not be revealed to or be reconstructible by any adversary.

For vector-valued inputs, one can apply the solution of Problems 1-4 in each dimension. 


\section{Dynamic average consensus}

In this section, we introduce a distributed dynamic average consensus algorithm that solves Problem 1 with a steady-state error for arbitrary time-varying input signals. We show that the size of this error can be controlled using a design parameter and that, for special classes of inputs, the steadystate error is zero. We also analyze the asymptotic correctness of the algorithm under time-varying interaction topologies and characterize the requirements on the stepsize for discrete-time implementations.

\subsection{Fixed interaction topology}

Here, we assume that the interaction topology of the network is fixed. We propose the following distributed algorithm as our solution for Problem 1

$$
\begin{aligned}
& \dot{x}^{i}=\dot{u}^{i}-\alpha\left(x^{i}-u^{i}\right)-\beta \sum_{j=1}^{N} \mathbf{L}_{i j} x^{j}-v^{i}, \\
& \dot{v}^{i}=\alpha \beta \sum_{j=1}^{N} \mathbf{L}_{i j} x^{j}
\end{aligned}
$$

where for $i \in\{1, \ldots, N\}, x^{i}, v^{i} \in \mathbb{R}$ are variables associated with agent $i$. Also, $\mathbf{L}$ is the Laplacian of the digraph $\mathcal{G}$ modeling the interaction topology. This algorithm uses the last two terms of (4a) as a proportional integral feedback to impose agreement among neighboring agents while these agents, because of the first two terms of (4a), are moving towards their respective input signal. Under suitable conditions on the communication topology, explained below, this scheme results in each agents eventually following the average of all the inputs across the network. The constants $\alpha, \beta \in \mathbb{R}$

are design parameters that can be used to tune the algorithm performance. In the following, we study the convergence and stability properties by using the equivalent compact form below

$$
\begin{aligned}
& \dot{\boldsymbol{y}}=-\alpha \boldsymbol{y}-\beta \mathbf{L} \boldsymbol{y}-\boldsymbol{w}, \\
& \dot{\boldsymbol{w}}=\alpha \beta \mathbf{L} \boldsymbol{y}-\boldsymbol{\Pi}_{N}(\ddot{\boldsymbol{u}}+\alpha \dot{\boldsymbol{u}}) .
\end{aligned}
$$


where

$$
\begin{aligned}
& y^{i}=x^{i}-\frac{1}{N} \sum_{j=1}^{N} u^{j}, \quad i \in\{1, \ldots, N\} \\
& \boldsymbol{w}=\boldsymbol{v}-\overline{\boldsymbol{v}}, \quad \overline{\boldsymbol{v}}=\boldsymbol{\Pi}_{N}(\dot{\boldsymbol{u}}+\alpha \boldsymbol{u}) .
\end{aligned}
$$

Recall from Section 3 that $x^{i}$ is the agreement state of agent $i$. Thus, with the change of variables (6a) we are transferring the desired equilibrium of the system, in agreement state, to zero. We start our study by analyzing the stability and convergence properties of the zero-system of (5), i.e.,

$$
\left[\begin{array}{c}
\dot{\boldsymbol{y}} \\
\dot{\boldsymbol{w}}
\end{array}\right]=\boldsymbol{A}\left[\begin{array}{c}
\boldsymbol{y} \\
\boldsymbol{w}
\end{array}\right], \quad \text { where } \boldsymbol{A}=\left[\begin{array}{cc}
-\alpha \boldsymbol{I}_{N}-\beta \mathbf{L} & -\boldsymbol{I}_{N} \\
\alpha \beta \mathbf{L} & \mathbf{0}
\end{array}\right] .
$$

In the following, we show that the dynamical system (7), over a strongly connected and weightbalanced digraph, is stable and convergent.

Lemma 4.1 (Asymptotic convergence of (7)): Let $\mathcal{G}$ be strongly connected and weight-balanced. For any $\alpha, \beta>0$, the trajectory of (7) over $\mathcal{G}$ starting from any initial condition $\boldsymbol{y}(0), \boldsymbol{w}(0) \in \mathbb{R}^{N}$ satisfies,

$$
y^{i}(t) \rightarrow-\frac{\alpha^{-1}}{N} \sum_{j=1}^{N} w^{j}(0), \quad w^{i}(t) \rightarrow \frac{1}{N} \sum_{j=1}^{N} w^{j}(0), \quad \text { as } t \rightarrow \infty, \quad \forall i \in\{1, \ldots, N\},
$$

exponentially fast with a rate of convergence upper bounded by $\min \left\{\alpha, \beta \Re\left(\lambda_{2}\right)\right\}$.

Proposition 4.1 Consider the following change of variables where $\boldsymbol{r}=\frac{1}{\sqrt{N}} \mathbf{1}_{N}$ and $\boldsymbol{R}$ is such that $\boldsymbol{r}^{\top} \boldsymbol{R}=0$ and $\boldsymbol{R}^{\top} \boldsymbol{R}=\boldsymbol{I}_{N-1}$,

$$
\left[\begin{array}{l}
\boldsymbol{p} \\
\boldsymbol{q}
\end{array}\right]=\boldsymbol{T}_{1} \boldsymbol{T}_{2}\left[\begin{array}{l}
\boldsymbol{y} \\
\boldsymbol{w}
\end{array}\right], \quad \boldsymbol{T}_{1}=\left[\begin{array}{cc}
\boldsymbol{I}_{N} & \mathbf{0} \\
\alpha \boldsymbol{I}_{N} & \boldsymbol{I}_{N}
\end{array}\right], \quad \boldsymbol{T}_{2}=\left[\begin{array}{cc}
\boldsymbol{T}_{3}^{\top} & \mathbf{0} \\
\mathbf{0} & \boldsymbol{T}_{3}^{\top}
\end{array}\right], \quad \boldsymbol{T}_{3}=\left[\begin{array}{ll}
\boldsymbol{r} & \boldsymbol{R}
\end{array}\right] .
$$

We partition the new variables as $\boldsymbol{p}=\left(p_{1}, \boldsymbol{p}_{2: N}\right)$ and $\boldsymbol{q}=\left(q_{1}, \boldsymbol{q}_{2: N}\right)$, where $p_{1}, q_{1} \in \mathbb{R}$ and $\boldsymbol{p}_{2: N}, \boldsymbol{q}_{2: N} \in$ 
$\mathbb{R}^{N-1}$. Using (9) the dynamics (7) can be stated in the following equivalent form

$$
\begin{aligned}
{\left[\begin{array}{c}
\dot{p}_{1} \\
\dot{q}_{1}
\end{array}\right] } & =\widetilde{\boldsymbol{A}}\left[\begin{array}{c}
p_{1} \\
q_{1}
\end{array}\right], \quad \widetilde{\boldsymbol{A}}=\left[\begin{array}{cc}
0 & -1 \\
0 & -\alpha
\end{array}\right], \\
{\left[\begin{array}{c}
\dot{\boldsymbol{p}}_{2: N} \\
\dot{\boldsymbol{q}}_{2: N}
\end{array}\right] } & =\overline{\boldsymbol{A}}\left[\begin{array}{c}
\boldsymbol{p}_{2: N} \\
\boldsymbol{q}_{2: N}
\end{array}\right], \quad \overline{\boldsymbol{A}}=\left[\begin{array}{cc}
-\beta \boldsymbol{R}^{\top} \mathbf{L} \boldsymbol{R} & -\boldsymbol{I}_{N-1} \\
\mathbf{0} & -\alpha \boldsymbol{I}_{N-1}
\end{array}\right] .
\end{aligned}
$$

The eigenvalues of $\widetilde{\boldsymbol{A}}$ are 0 and $-\alpha$. The eigenvalues of the matrix $\overline{\boldsymbol{A}}$ are $-\alpha$, with multiplicity $N-1$, and $-\beta \lambda_{i}$, with $i \in\{2, \ldots, N\}$. Recall that $\lambda_{i}$ 's are eigenvalues of $\mathbf{L}$. For a strongly connected digraph, $\lambda_{1}=0$ and the rest of the eigenvalues have positive real parts. Therefore, for $\alpha, \beta>0$, the dynamical system (10), and equivalently (7), is a stable linear system.

The null-space of the system matrix $\boldsymbol{A}$ is spanned by $\left(\mathbf{1}_{N},-\alpha \mathbf{1}_{N}\right)$, the eigenvector associated with zero eigenvalue. Therefore, (7) converges exponentially fast to the set

$$
\left\{(\boldsymbol{y}, \boldsymbol{w}) \mid \boldsymbol{y}=\mu \mathbf{1}_{N}, \boldsymbol{w}=-\mu \alpha \mathbf{1}_{N}, \quad \mu \in \mathbb{R}\right\} .
$$

Left multiplying both sides of (7) by $\operatorname{Diag}\left(\mathbf{0}_{N}^{\top}, \mathbf{1}_{N}^{\top}\right)$ and invoking the weight-balanced property of the digraph, we obtain $\sum_{i=1}^{N} \dot{w}^{i}=0$, and therefore,

$$
\sum_{i=1}^{N} w^{i}(t)=\sum_{i=1}^{N} w^{i}(0), \quad \forall t \geq 0
$$

The combination of (11) and (12) yields that, from any initial condition $\boldsymbol{y}(0), \boldsymbol{w}(0) \in \mathbb{R}^{N}$, the trajectory of the dynamical system (7) satisfies (8), exponentially fast. Based on (2), the rate of convergence is $\min \left\{\alpha, \beta \Re\left(\lambda_{2}\right)\right\}$.

The next result further probes into the properties of the dynamical system (7) by upper bounding the difference between the state $y^{i}$ of agent $i$ at any time $t$ and the equilibrium value. This bound is instrumental later in the characterization of the steady-state error of (4).

Lemma 4.2 (Upper bound on trajectories of (7)): Under the assumptions of Lemma 4.1, the following bound holds for each $i \in\{1, \ldots, N\}$,

$$
\left|y^{i}(t)+\frac{\alpha^{-1}}{N} \sum_{j=1}^{N} w^{j}(0)\right| \leq\left\|\boldsymbol{y}(t)+\alpha^{-1} \boldsymbol{r} \boldsymbol{r}^{\top} \boldsymbol{w}(0)\right\| \leq s(t),
$$


where

$$
\begin{aligned}
s(t)= & \left(\mathrm{e}^{-\alpha t}+\mathrm{e}^{-\beta \hat{\lambda}_{2} t}\right)\|\boldsymbol{y}(0)\|+\alpha^{-1} \mathrm{e}^{-\alpha t}\|\boldsymbol{w}(0)\| \\
& + \begin{cases}\left(\beta \hat{\lambda}_{2}-\alpha\right)^{-1}\left(\mathrm{e}^{-\alpha t}-\mathrm{e}^{-\beta \hat{\lambda}_{2} t}\right)(\alpha\|\boldsymbol{y}(0)\|+\|\boldsymbol{w}(0)\|), & \text { if } \alpha \neq \beta \hat{\lambda}_{2}, \\
t \mathrm{e}^{-\beta \hat{\lambda}_{2} t}(\alpha\|\boldsymbol{y}(0)\|+\|\boldsymbol{w}(0)\|), & \text { if } \alpha=\beta \hat{\lambda}_{2} .\end{cases}
\end{aligned}
$$

Proposition 4.2 The solution of the state equation (10) from any initial condition $\boldsymbol{y}(0), \boldsymbol{w}(0) \in \mathbb{R}^{N}$ is $\left(p_{1}(t), q_{1}(t), \boldsymbol{p}_{2: N}(t), \boldsymbol{q}_{2: N}(t)\right)=\boldsymbol{\Omega}(t)\left(p_{1}(0), q_{1}(0), \boldsymbol{p}_{2: N}(0), \boldsymbol{q}_{2: N}(0)\right)$, where

$$
\boldsymbol{\Omega}(t)=\left[\begin{array}{cccc}
1 & \alpha^{-1}\left(\mathrm{e}^{-\alpha t}-1\right) & 0 & 0 \\
0 & \mathrm{e}^{-\alpha t} & 0 & 0 \\
\mathbf{0} & \mathbf{0} & \boldsymbol{\Phi}(t, 0) & -\int_{0}^{t} \boldsymbol{\Phi}(t, \tau) \mathrm{e}^{-\alpha \tau} d \tau \\
\mathbf{0} & \mathbf{0} & \mathbf{0} & \mathrm{e}^{-\alpha t} \boldsymbol{I}_{N-1}
\end{array}\right],
$$

and $\boldsymbol{\Phi}(t, \tau)=\mathrm{e}^{-\beta \boldsymbol{R}^{\top} \mathbf{L} \boldsymbol{R}(t-\tau)}$. Now, from [22, Fact 11.15.7, item xvii], we deduce

$$
\|\boldsymbol{\Phi}(t, \tau)\|=\left\|\mathrm{e}^{-\beta \boldsymbol{R}^{\top} \mathbf{L} \boldsymbol{R}(t-\tau)}\right\| \leq \mathrm{e}^{-\beta \hat{\lambda}_{2}(t-\tau)}
$$

and hence

$$
\left\|\int_{0}^{t} \boldsymbol{\Phi}(t, \tau) \mathrm{e}^{-\alpha \tau} d \tau\right\| \leq \int_{0}^{t} \mathrm{e}^{-\beta \hat{\lambda}_{2}(t-\tau)} \mathrm{e}^{-\alpha \tau} d \tau
$$

Now, using the change of variables (9), one has

$$
\boldsymbol{y}(t)=\boldsymbol{S}_{11} \boldsymbol{y}(0)+\boldsymbol{S}_{12} \boldsymbol{w}(0)
$$

where

$$
\begin{aligned}
& \boldsymbol{S}_{11}=\mathrm{e}^{-\alpha t} \boldsymbol{r} \boldsymbol{r}^{\top}+\boldsymbol{R} \boldsymbol{\Phi}(t, 0) \boldsymbol{R}^{\top}-\alpha \boldsymbol{R}\left(\int_{0}^{t} \boldsymbol{\Phi}(t, \tau) \mathrm{e}^{-\alpha \tau} d \tau\right) \boldsymbol{R}^{\top} \\
& \boldsymbol{S}_{12}=\left(-\alpha^{-1}+\alpha^{-1} \mathrm{e}^{-\alpha t}\right) \boldsymbol{r} \boldsymbol{r}^{\top}-\boldsymbol{R}\left(\int_{0}^{t} \boldsymbol{\Phi}(t, \tau) \mathrm{e}^{-\alpha \tau} d \tau\right) \boldsymbol{R}^{\top}
\end{aligned}
$$

The result now follows from using (15) and (16) to bound the expression (17).

Next, using the results guaranteed by Lemma 4.2 we study the convergence and stability properties of our proposed dynamic average consensus algorithm (4). We start by establishing an upper bound on its tracking error for any given initial condition. 
Theorem 4.1 (Upper bound on the tracking error of (4)): Let $\mathcal{G}$ be strongly connected and weightbalanced. Each agent has a piecewise continuously differentiable input $u^{i}(t)$. For $\alpha, \beta>0$, the trajectory of the algorithm (4) over $\mathcal{G}$ starting from any initial condition $\boldsymbol{x}(0), \boldsymbol{v}(0) \in \mathbb{R}^{N}$ satisfies, for all $i \in\{1, \ldots, N\}$,

$$
\begin{aligned}
\left|x^{i}(t)-\frac{1}{N} \sum_{j=1}^{N} u^{j}(t)+\frac{\alpha^{-1}}{N} \sum_{j=1}^{N} v^{j}(0)\right| \leq & s(t)+\int_{0}^{t} \mathrm{e}^{-\beta \hat{\lambda}_{2}(t-\tau)}\left\|\boldsymbol{\Pi}_{N} \dot{\boldsymbol{u}}(\tau)\right\| d \tau+ \\
& \begin{cases}\left(\beta \hat{\lambda}_{2}-\alpha\right)^{-1}\left(\mathrm{e}^{-\alpha t}-\mathrm{e}^{-\beta \hat{\lambda}_{2} t}\right)\|\dot{\boldsymbol{u}}(0)\|, & \text { if } \alpha \neq \beta \hat{\lambda}_{2}, \\
t \mathrm{e}^{-\beta \hat{\lambda}_{2} t}\|\dot{\boldsymbol{u}}(0)\|, & \text { if } \alpha=\beta \hat{\lambda}_{2},\end{cases}
\end{aligned}
$$

where $s(t)$ is defined in (13), and $\boldsymbol{y}$ and $\boldsymbol{w}$ are defined in (6).

Proposition 4.3 Using the change of the variables (9) we can represent (5), an equivalent representation of (4), in the following equivalent form where $\widetilde{\boldsymbol{A}}$ and $\overline{\boldsymbol{A}}$ are defined in (10),

$$
\begin{aligned}
& {\left[\begin{array}{c}
\dot{p}_{1} \\
\dot{q}_{1}
\end{array}\right]=\widetilde{\boldsymbol{A}}\left[\begin{array}{c}
p_{1} \\
q_{1}
\end{array}\right],} \\
& {\left[\begin{array}{c}
\dot{\boldsymbol{p}}_{2: N} \\
\dot{\boldsymbol{q}}_{2: N}
\end{array}\right]=\overline{\boldsymbol{A}}\left[\begin{array}{l}
\boldsymbol{p}_{2: N} \\
\boldsymbol{q}_{2: N}
\end{array}\right]-\left[\begin{array}{c}
\mathbf{0} \\
\boldsymbol{R}^{\top}
\end{array}\right](\ddot{\boldsymbol{u}}+\alpha \dot{\boldsymbol{u}}),}
\end{aligned}
$$

For any given initial conditions, the solution of the state equation (20) is

$$
\left[\begin{array}{c}
p_{1}(t) \\
q_{1}(t) \\
\boldsymbol{p}_{2: N}(t) \\
\boldsymbol{q}_{2: N}(t)
\end{array}\right]=\boldsymbol{\Omega}(t)\left[\begin{array}{c}
p_{1}(0) \\
q_{1}(0) \\
\boldsymbol{p}_{2: N}(0) \\
\boldsymbol{q}_{2: N}(0)
\end{array}\right]-\left[\begin{array}{c}
0 \\
\int_{0}^{t} \boldsymbol{\Phi}(t, \tau) \mathrm{e}^{-\alpha \tau} d \tau\left(\boldsymbol{q}_{2: N}(0)+\boldsymbol{R}^{\top} \dot{\boldsymbol{u}}(0)\right)-\int_{0}^{t} \boldsymbol{\Phi}(t, \tau) \boldsymbol{R}^{\top} \dot{\boldsymbol{u}}(\tau) d \tau \\
-\boldsymbol{R}^{\top} \dot{\boldsymbol{u}}(0)+\boldsymbol{R}^{\top} \dot{\boldsymbol{u}}(t)
\end{array}\right],
$$

where $\boldsymbol{\Omega}(t)$ is defined in (14). Recalling the change of variables (9), we have

$$
\boldsymbol{y}(t)=\boldsymbol{S}_{11} \boldsymbol{y}(0)+\boldsymbol{S}_{12} \boldsymbol{w}(0)-\boldsymbol{R} \int_{0}^{t} \boldsymbol{\Phi}(t, \tau) \mathrm{e}^{-\alpha \tau} d \tau \boldsymbol{R}^{\top} \dot{\boldsymbol{u}}(0)+\boldsymbol{R} \int_{0}^{t} \boldsymbol{\Phi}(t, \tau) \boldsymbol{R}^{\top} \dot{\boldsymbol{u}}(\tau) d \tau,
$$

where $\boldsymbol{S}_{11}$ and $\boldsymbol{S}_{12}$ are defined in (18). Note that (6b) implies that $\sum_{i=1}^{N} w^{i}(0)=\sum_{i=1}^{N} v^{i}(0)$. Notice also that $\boldsymbol{R}^{\top}=\boldsymbol{R}^{\top} \boldsymbol{\Pi}_{N}$, and $\|\boldsymbol{R}\|=\left\|\boldsymbol{R}^{\top}\right\|=\sigma_{\max }(\boldsymbol{R})=1$. Then, by recalling (15), it is straightforward to show that (19) is satisfied. 
The next result shows that, for input signals whose orthogonal projection into the agreement space are essentially bounded, the algorithm (4) solves Problem 1 with a bounded steady-state error.

Corollary 4.1 (The algorithm (4) solves Problem 1): Let $\mathcal{G}$ be strongly connected and weightbalanced. Assume that the derivatives of the inputs of the network satisfy $\left\|\boldsymbol{\Pi}_{N} \dot{\boldsymbol{u}}\right\|_{\text {ess }}=\gamma<\infty$. Then, for any $\alpha, \beta>0$ the algorithm (4) over $\mathcal{G}$ initialized at $x^{i}(0), v^{i}(0) \in \mathbb{R}$ such that $\sum_{i=1}^{N} v^{i}(0)=0$ solves Problem 1 with an upper-bounded steady-state error. Specifically,

$$
\lim _{t \rightarrow \infty} \sup \left|x^{i}(t)-\frac{1}{N} \sum_{j=1}^{N} u^{j}(t)\right| \leq\left(\beta \hat{\lambda}_{2}\right)^{-1} \gamma, \quad i \in\{1, \ldots, N\} .
$$

Proposition 4.4 In Theorem 4.1, for a strongly connected and weight-balanced digraph, we showed that the trajectories of the algorithm (4), for any $x^{i}(0), v^{i}(0) \in \mathbb{R}, i \in\{1, \ldots, N\}$, satisfy the bound (19). Then, we can easily deduce (22) from (19) using

$$
\int_{0}^{t} \mathrm{e}^{-\beta \hat{\lambda}_{2}(t-\tau)}\left\|\boldsymbol{\Pi}_{N} \dot{\boldsymbol{u}}(\tau)\right\| d \tau \leq\left(\beta \hat{\lambda}_{2}\right)^{-1}\left(1-\mathrm{e}^{-\beta \hat{\lambda}_{2} t}\right) \gamma
$$

Remark 4.1 (Effect of faulty initial conditions): The condition $\sum_{i=1}^{N} v^{i}(0)=0$ of Corollary 4.1 can be easily satisfied if each agent starts at $v^{i}(0)=0$. This is a mild requirement because $v^{i}$ is an internal state for agent $i$, and therefore it is not affected by imperfect communication errors. Additionally, for large networks, if we assume that the initialization error is zero-mean Gaussian noise, we can expect $\sum_{i=1}^{N} v^{i}(0)=0$.

Remark 4.2 (Tuning the performance of (4) via design parameters): Corollary 4.1 shows that to reduce the nonzero steady-state error, one can either increase the graph connectivity (larger $\hat{\lambda}_{2}$ ) or use a larger value of $\beta$. The parameter $\alpha$ can also be exploited to regulate the algorithm performance. According to the bound (19) the rate of convergence of the transient behavior is governed by $\min \left\{\alpha, \beta \hat{\lambda}_{2}\right\}$. If one is forced to use large $\beta \hat{\lambda}_{2}$ to reduce the steady-state error, then $\alpha$ can fulfill the role of regulating the rate of convergence of the algorithm.

Remark 4.3 (Comparison with input requirements of the solutions in the literature): In order to guarantee bounded steady-state tracking error, the solution we offer for Problem 1 through Corol- 
lary 4.1 only requires that the projection of the network's aggregated input derivative vector into the agreement space is bounded. This is more general than the requirements in the literature, which generally ask for bounded input and/or bounded derivatives (e.g., [4, 15, 17]).

In the following, we identify conditions involving the inputs and their derivatives under which the algorithm (4) solves Problem 1 with zero steady-state error.

Lemma 4.3 (Conditions on inputs for zero steady-state error of (4)): Let $\mathcal{G}$ be strongly connected and weight-balanced. Assume there exists $\alpha>0$ such that, for all $i \in\{1, \ldots, N\}$, one of the following conditions are satisfied

(a) $\dot{u}^{i}(t)+\alpha u^{i}(t)$ converges to a common function $l(t)$ as $t \rightarrow \infty$;

(b) $\ddot{u}^{i}(t)+\alpha \dot{u}^{i}(t)$ converges to a common function $l(t)$ as $t \rightarrow \infty$.

Then, the algorithm (4) over $\mathcal{G}$ with the given $\alpha$, and $x^{i}(0), v^{i}(0) \in \mathbb{R}$ such that $\sum_{i=1}^{N} v^{i}(0)=0$, for any $\beta>0$, makes $x^{i}(t) \rightarrow \frac{1}{N} \sum_{j=1}^{N} u^{j}(t)$ as $t \rightarrow \infty$, for all $i \in\{1, \ldots, N\}$.

Proposition 4.5 Using the change of variables (6a) we can represent (4) in the following equivalent compact form

$$
\begin{aligned}
& \dot{\boldsymbol{y}}=-\alpha \boldsymbol{y}-\beta \mathbf{L} \boldsymbol{y}-\boldsymbol{v}+\boldsymbol{\Pi}_{N}(\dot{\boldsymbol{u}}+\alpha \boldsymbol{u}), \\
& \dot{\boldsymbol{v}}=\alpha \beta \mathbf{L} \boldsymbol{y} .
\end{aligned}
$$

When condition (a) holds we have $\boldsymbol{\Pi}_{N}(\dot{\boldsymbol{u}}+\alpha \boldsymbol{u}) \rightarrow \mathbf{0}$, as $t \rightarrow \infty$. Then, (23) is a linear system with a vanishing input $\boldsymbol{\Pi}_{N}(\dot{\boldsymbol{u}}+\alpha \boldsymbol{u})$. Therefore, it converges to the equilibrium of its zero-system. In light of Lemma 4.1, we conclude that $y^{i}(t) \rightarrow-\frac{\alpha^{-1}}{N} \sum_{j=1}^{N} v^{j}(0)$ asymptotically for all $i \in\{1, \ldots, N\}$. However, due to initialization requirement we have $\sum_{i=1}^{N} v^{i}(0)=0$. As a result $x^{i}(t) \rightarrow \frac{1}{N} \sum_{j=1}^{N} u^{j}(t)$ globally asymptotically for $i \in\{1, \ldots, N\}$.

When condition (b) holds we have $\boldsymbol{\Pi}_{N}(\ddot{\boldsymbol{u}}+\alpha \dot{\boldsymbol{u}}) \rightarrow \mathbf{0}$, as $t \rightarrow \infty$. Recall (5) the equivalent representation of (4). It is a linear system with a vanishing input $\boldsymbol{\Pi}_{N}(\dot{\boldsymbol{u}}+\alpha \boldsymbol{u})$. Then, using a 
similar argument used for (23) above, we can show that in (5) $y^{i}(t) \rightarrow-\frac{\alpha^{-1}}{N} \sum_{j=1}^{N} w^{j}(0)$ asymptotically for all $i \in\{1, \ldots, N\}$. Using (6b), we can show $\sum_{i=1}^{N} w^{i}(0)=\sum_{i=1}^{N} v^{i}(0)$. As a result $x^{i}(t) \rightarrow \frac{1}{N} \sum_{j=1}^{N} u^{j}(t)$ globally asymptotically for $i \in\{1, \ldots, N\}$.

Remark 4.4 (Inputs that satisfy the conditions of Lemma 4.3): The classes of inputs in Lemma 4.3 depend on the parameter $\alpha$ which must be known by each agent in order to obtain zero steady-state error. There are classes of inputs that satisfy the conditions regardless of the value of $\alpha$, such as static inputs and dynamic inputs which differ from one another by static values. For these classes of inputs, $\boldsymbol{\Pi}_{N}(\ddot{\boldsymbol{u}}+\alpha \dot{\boldsymbol{u}})=\mathbf{0}$, and the convergence is exponential with rate $\min \left\{\alpha, \beta \Re\left(\lambda_{2}\right)\right\}$.

\subsection{Time-varying interaction topologies}

In this section, we analyze the stability and convergence properties of the dynamic average consensus algorithm (4) over networks with changing interaction topology. Changes can be due to unreliable transmission, limited communication/sensing range, or obstacles. Let $(\mathcal{V}, \mathcal{E}(t), \mathbf{A}(t))$ be a timevarying digraph, where the nonzero entries of the adjacency matrix are uniformly lower and upper bounded (i.e., $a_{i j}(t) \in[\underline{a}, \bar{a}]$, where $0<\underline{a} \leq \bar{a}$, if $(j, i) \in \mathcal{E}(t)$, and $a_{i j}=0$ otherwise). Intuitively one can expect that consensus in switching networks will occur if there is occasional enough flow of information from every node in the network to every other node. Then, according to Section 2.3, in order to describe our switching network model, we start by specifying the set of admissible switching signals.

Definition 1 (Admissible switching set $\mathcal{S}_{\text {admis }}$ ): An admissible switching set $\mathcal{S}_{\text {admis }}$ is a set of piecewise constant switching signals $\sigma:[0, \infty) \rightarrow \mathcal{P}$ with some dwell time $t_{L}$ (i.e., $t_{k+1}-t_{k}>t_{L}>0$, for all $k=0,1, \ldots)$ such that

- the induced digraph $\Gamma\left(\mathbf{A}_{\sigma(t)}\right)$ is weight-balanced for $t \geq t_{0}$;

- the number of contiguous, nonempty, uniformly bounded time-intervals $\left[t_{i_{j}}, t_{i_{j+1}}\right), j=1,2, \ldots$, starting at $t_{i_{1}}=t_{0}$, with the property that $\cup_{t_{i_{j}}}^{t_{i_{j+1}}} \Gamma\left(\mathbf{A}_{\sigma(t)}\right)$ is a jointly strongly connected digraph 
goes to infinity as $t \rightarrow \infty$.

Our model of network with switching topology is then $\Gamma\left(\mathbf{A}_{\sigma}\right)$, with $\sigma \in \mathcal{S}_{\text {admis }}$. The algorithm (4), after applying the change of variables (6), is represented in compact form as follows

$$
\left[\begin{array}{c}
\dot{\boldsymbol{y}} \\
\dot{\boldsymbol{w}}
\end{array}\right]=\boldsymbol{A}_{\sigma(t)}\left[\begin{array}{l}
\boldsymbol{y} \\
\boldsymbol{w}
\end{array}\right]-\left[\begin{array}{c}
\mathbf{0} \\
\boldsymbol{\Pi}_{N}(\ddot{\boldsymbol{u}}+\alpha \dot{\boldsymbol{u}})
\end{array}\right], \quad \boldsymbol{A}_{\sigma(t)}=\left[\begin{array}{cc}
-\alpha \boldsymbol{I}_{N}-\beta \mathbf{L}_{\sigma(t)} & -\boldsymbol{I}_{N} \\
\alpha \beta \mathbf{L}_{\sigma(t)} & \mathbf{0}
\end{array}\right] .
$$

Similarly to our analysis of the algorithm over fixed interaction topologies, we start by examining the zero-system of (24), i.e.,

$$
\left[\begin{array}{c}
\dot{\boldsymbol{y}} \\
\dot{\boldsymbol{w}}
\end{array}\right]=\boldsymbol{A}_{\sigma(t)}\left[\begin{array}{l}
\boldsymbol{y} \\
\boldsymbol{w}
\end{array}\right]
$$

The following result analyzes the convergence and stability properties of the switched dynamical system (25) when the switching signal $\sigma \in \mathcal{S}_{\text {admis }}$.

Lemma 4.4 (Asymptotic convergence of (25)): Let $\sigma \in \mathcal{S}_{\text {admis }}$ and consider $\mathcal{G}(t)=\Gamma\left(\mathbf{A}_{\sigma(t)}\right)$ for $t \geq 0$. Then, for any $\alpha, \beta>0$, the trajectory of the algorithm (25) starting from any initial condition $\boldsymbol{y}(0), \boldsymbol{w}(0) \in \mathbb{R}^{N}$ satisfies (8), exponentially fast.

Proposition 4.6 Using the change of the variables (9), we can represent (25) in the equivalent form (10) in which $\overline{\boldsymbol{A}}$ and $\mathbf{L}$ are replaced by $\overline{\boldsymbol{A}}_{\sigma(t)}$ and $\mathbf{L}_{\sigma(t)}$, respectively. We can write $\dot{\boldsymbol{p}}$ as follows

$$
\dot{p}=-\boldsymbol{T}_{3}^{\top} \mathbf{L}_{\sigma} \boldsymbol{T}_{3} \boldsymbol{p}-\boldsymbol{q}
$$

We can look at this dynamical equation as a linear system with input $\boldsymbol{q}$ which vanishes exponentially fast (notice that $\dot{\boldsymbol{q}}=-\alpha \boldsymbol{q}$ ). Next, we examine the stability of zero-system of (26). Under the state transformation $\boldsymbol{\eta}=\boldsymbol{T}_{3} \boldsymbol{p}$, this zero-system can be represented in the following equivalent form

$$
\dot{\boldsymbol{\eta}}=-\mathbf{L}_{\sigma} \boldsymbol{\eta}
$$

According to [9, Theorem 2.33], when the switching signal $\sigma$ is such that the number of contiguous, nonempty, uniformly bounded time-intervals $\left[t_{i_{j}}, t_{i_{j+1}}\right), j=1,2, \ldots$, starting at $t_{i_{1}}=t_{0}$, with the 
property that $\cup_{t_{i_{j}}}^{t_{i_{j+1}}} \Gamma\left(\mathbf{A}_{\sigma(t)}\right)$ has a spanning tree, then (27) asymptotically achieves consensus. Invoking this result, we can conclude that for $\sigma \in \mathcal{S}_{\text {admis }}$, the trajectories of (27) converge asymptotically to $\frac{1}{N} \sum_{j=1}^{N} \eta_{j}(0)$ where $\eta_{i}(0)$ is the ith element of $\boldsymbol{\eta}(0)$. For zero-system of $(26)$, this is equivalent to $p_{1}(t) \rightarrow p_{1}(0)$ and $\boldsymbol{p}_{2: N}(t) \rightarrow \mathbf{0}$ uniformly asymptotically for all $\sigma \in \mathcal{S}_{\text {admis. }}$ The switching signal $\sigma \in \mathcal{S}_{\text {admis }}$ is a trajectory-independent (it is time-dependent) switching signal. Then, Lemma 2.1 implies that the convergence of the zero system of (26) is indeed globally uniformly exponentially fast. Using input-to-state stability results (see [23, 24]), then we can conclude that in $(26), p_{1}(t) \rightarrow p_{1}(0)$ and $\boldsymbol{p}_{2: N}(t) \rightarrow \mathbf{0}$ as $t \rightarrow \infty$ uniformly globally exponentially. Recall the change of variable (9), then it is easy to show that for (25) we also have (8).

Obtaining an explicit value for the rate of convergence of (25) for all possible $\sigma \in \mathcal{S}_{\text {admis }}$ is not straightforward. However, we can show that the rate of convergence is upper bounded by $\max _{p \in \mathcal{P}}\left(\Re\left(\lambda_{p 2}\right)\right)$, where $\lambda_{p 2}$ is the eigenvalue of $\mathbf{L}_{p}$ with smallest nonzero real part. The following result relates the upper bound on the difference between the state $y^{i}(t)$ of agent $i$ at any time $t$ and the final agreement value to the rate of convergence of (8).

Lemma 4.5 (Upper bound on trajectories of (25)): Under the assumptions of Lemma 4.4, the following bound holds for each $i \in\{1, \cdots, N\}$,

$$
\left|y^{i}(t)+\frac{\alpha^{-1}}{N} \sum_{i=1}^{N} w^{i}(0)\right| \leq\left\|\boldsymbol{y}_{\mathrm{T}}(t)+\alpha^{-1} \boldsymbol{r} \boldsymbol{r}^{\top} \boldsymbol{w}_{\mathrm{T}}(0)\right\| \leq \hat{s}(t),
$$

where $\hat{s}(t)$ is the same as $s(t)$ in (13) only $\hat{\lambda}_{2}$ is replaced by $\hat{\lambda}_{\sigma}>0$ where $\hat{\lambda}_{\sigma}$ satisfies

$$
\left\|\mathrm{e}^{-\beta \boldsymbol{R}^{\top} \mathbf{L}_{\sigma(t)} \boldsymbol{R}\left(t-t_{0}\right)}\right\| \leq \kappa \mathrm{e}^{-\beta \hat{\lambda}_{\sigma}\left(t-t_{0}\right)}, \quad \forall t \geq t_{0} \geq 0
$$

for some finite $0<\kappa$.

Proposition 4.7 We follow the same steps of the proof of Lemma 4.2. The only difference is that the norm bound (15) of the transition matrix of $\dot{\boldsymbol{p}}_{2: N}$ state equation has to be modified, as explained below. We showed in the proof of Lemma 4.5 that when $\sigma \in \mathcal{S}_{\text {admis }}$ for all $t \geq t_{0}$, the zero-system 
of (26) is exponentially stable. Therefore, there exist positive $\hat{\lambda}_{\sigma}$ and $\kappa$ such that

$$
\left\|\Phi\left(t, t_{0}\right)=\mathrm{e}^{-\beta \boldsymbol{R}^{\top} \mathbf{L}_{\sigma(t)} \boldsymbol{R}\left(t-t_{0}\right)}\right\| \leq \kappa \mathrm{e}^{-\beta \hat{\lambda}_{\sigma}\left(t-t_{0}\right)}, \quad \forall t \geq t_{0} \geq 0
$$

As a result, in the case of switched dynamical systems, in (16) $\hat{\lambda}_{2}$ is replaced by $\hat{\lambda}_{\sigma}$. Then, from (17) we can deduce the bound (28).

In light of Lemma 4.5, the extension of the results on the stability analysis and ultimate convergence error bound of the algorithm (4) over fixed interaction topologies to switching networks whose switching signal $\sigma \in \mathcal{S}_{\text {admis }}$ is straightforward. For such switching networks, Theorem 4.1 and Corollary 4.1 are valid, with the only change of replacing $\beta \hat{\lambda}_{2}$ by $\beta \hat{\lambda}_{\sigma}$, cf. (29), in the statement. Because of Lemma 4.4, the proof that Lemma 4.3 applies to switched networks with $\sigma \in \mathcal{S}_{\text {admis }}$ is straightforward. For the sake of brevity the detailed statements and proofs are omitted.

\subsection{Discrete-time implementation over fixed interaction topologies}

Here, we study a discrete-time algorithm that solves Problem 1 with non-zero steady-state error. In doing so, we are motivated by the aim of understanding the differences and connections between continuous- and discrete-time systems for multi-agent systems and by practical considerations regarding algorithm implementability. Given a stepsize $\delta>0$, for $i \in\{1, \ldots, N\}$, consider

$$
\begin{aligned}
z^{i}(k+1) & =z^{i}(k)-\delta \alpha z^{i}(k)-\delta \beta \sum_{j=1}^{N} \mathbf{L}_{i j}\left(z^{j}(k)+u^{j}(k)\right)-\delta v^{i}(k), \\
v^{i}(k+1) & =v^{i}(k)+\delta \alpha \beta \sum_{j=1}^{N} \mathbf{L}_{i j}\left(z^{j}(k)+u^{j}(k)\right) \\
x^{i}(k) & =z^{i}(k)+u^{i}(k) .
\end{aligned}
$$

Using (30c) to obtain $z^{i}(k)=x^{i}(k)-u^{i}(k)$, and substituting this in (30a) and (30b), we obtain

$$
\begin{aligned}
& x^{i}(k+1)=x^{i}(k)-\delta \alpha\left(x^{i}(k)-u^{i}(k)\right)-\delta \beta \sum_{j=1}^{N} \mathbf{L}_{i j} x^{j}(k)-\delta v^{i}(k)+\Delta u^{i}(k), \\
& v^{i}(k+1)=v^{i}(k)+\delta \alpha \beta \sum_{j=1}^{N} \mathbf{L}_{i j} x^{j}(k),
\end{aligned}
$$


where $\Delta u^{i}(k)=u^{i}(k+1)-u^{i}(k)$. Notice that the discrete-time algorithm (30) is an equivalent iterative form of (4) obtained by Euler discretization with stepsize $\delta$. When $\delta \rightarrow 0$, we can expect that the stability and convergence properties of (30) are similar to that of (4), i.e., $x^{i}$ tracks the average of the network inputs in its $O\left(\beta^{-1}\right)$ neighborhood, provided the network topology is strongly connected and weight-balanced digraph. Notice that the structure (30) allows us to circumvent discretizing the derivative of the input signals and, as a result, avoid the one-step delayed tracking reported in [17]. Next, note that $u^{i}$ is never communicated directly.

Next, we explore the bounds on the stepsize $\delta$ such that (30) is convergent and tracks the input average. The proof of the results is presented in Appendix A. We start by studying the stability and convergence properties of the zero-system.

Lemma 4.6 (Convergence analysis and stepsize characterization of the zero-system of (31)): Let $\mathcal{G}$ be strongly connected and weight-balanced. For $\alpha, \beta>0$, the trajectory of the zero-system of discrete-time algorithm (31) over $\mathcal{G}$ starting from any initial condition $\boldsymbol{x}(0), \boldsymbol{v}(0) \in \mathbb{R}^{N}$ satisfies

$$
x^{i}(k) \rightarrow-\frac{\alpha^{-1}}{N} \sum_{i=1}^{N} v^{i}(0), \quad v^{i}(k) \rightarrow \frac{1}{N} \sum_{j=1}^{N} v^{j}(0), \quad \forall i \in\{1, \ldots, N\},
$$

asymptotically, as $k \rightarrow \infty$, provided $\delta \in\left(0, \min \left\{\alpha^{-1}, \beta^{-1}\left(d_{\max }^{\text {out }}\right)^{-1}\right\}\right)$.

The following result establishes an upper bound on the solutions of the algorithm (30) for any given initial conditions. In the following, we let $\boldsymbol{\Phi}(k, j)=\left(\boldsymbol{I}_{N-1}-\delta \beta \boldsymbol{R}^{\top} \mathbf{L} \boldsymbol{R}\right)^{k-j}$.

Theorem 4.2 (Upper bound on the tracking error of (31)): Let $\mathcal{G}$ be strongly connected and weightbalanced. Each agent has an input $u^{i}(k)$. For $\alpha, \beta>0$, the trajectory of the algorithm (30) over $\mathcal{G}$ 
starting from any initial condition $\boldsymbol{z}(0), \boldsymbol{v}(0) \in \mathbb{R}^{N}$ satisfies,

$$
\begin{aligned}
& \left|x^{i}(k)-\frac{1}{N} \sum_{j=1}^{N} u^{j}(k)+\frac{1}{N} \delta \sum_{j=0}^{k-1}(1-\delta \alpha)^{j} \sum_{j=1}^{N} v^{j}(0)\right| \leq\left\|\boldsymbol{y}(t)+\delta \sum_{j=0}^{k-1}(1-\delta \alpha)^{j} \boldsymbol{r} \boldsymbol{r}^{\top} \boldsymbol{w}(0)\right\| \leq \\
& \left|\left(1-\alpha \delta \sum_{j=0}^{k-1}(1-\delta \alpha)^{j}\right)\right|\|\boldsymbol{y}(0)\|+\|\boldsymbol{\Phi}(k, 0)\|\|\boldsymbol{y}(0)\|+\alpha\left\|\left(\sum_{j=0}^{k-1} \boldsymbol{\Phi}(k-1, j)(1-\delta \alpha)^{j}\right)\right\|\|\boldsymbol{y}(0)\|+ \\
& \left\|\left(\sum_{j=0}^{k-1} \boldsymbol{\Phi}(k-1, j)(1-\delta \alpha)^{j}\right)\right\|\|\boldsymbol{w}(0)\|+\left\|\sum_{j=0}^{k-1} \boldsymbol{\Phi}(k-1, j)(1-\delta \alpha)^{j}\right\|\|\Delta \boldsymbol{u}(0)\|+ \\
& \left\|\boldsymbol{R} \sum_{j=0}^{k-1} \boldsymbol{\Phi}(k-1, j) \boldsymbol{R}^{\top} \Delta \boldsymbol{u}(j)\right\|
\end{aligned}
$$

for all $i \in\{1, \ldots, N\}$, where $\boldsymbol{y}$ is defined in (6a) and $\boldsymbol{w}$ is

$$
\boldsymbol{w}=\boldsymbol{v}-\overline{\boldsymbol{v}}, \quad \overline{\boldsymbol{v}}=\boldsymbol{\Pi}_{N}(\Delta \boldsymbol{u}(k)+\delta \alpha \boldsymbol{u}(k))
$$

Next, we show that for networks with strongly connected and weight-balanced digraph topologies, the discrete-time algorithm (30) solves Problem 1 with a nonzero steady-state error, provided $\delta \in\left(0, \min \left\{\alpha^{-1}, \beta^{-1}\left(\mathrm{~d}_{\max }^{\text {out }}\right)^{-1}\right\}\right)$, the algorithm is initialized properly and the essential norm of the projection of the input difference vector into the agreement space is bounded.

Corollary 4.2 (The algorithm (30) solves Problem 1): Let $\mathcal{G}$ be strongly connected and weightbalanced. Assume that the differences of the inputs of the network satisfy $\left\|\boldsymbol{\Pi}_{N} \Delta \boldsymbol{u}\right\|_{\text {ess }}=\gamma<$ $\infty$. Then, for any $\alpha, \beta>0$, the algorithm (30) over $\mathcal{G}$ initialized at $z^{i}(0), v^{i}(0) \in \mathbb{R}$ such that $\sum_{i=1}^{N} v^{i}(0)=0$ solves Problem 1 (in the output $x^{i}$ ) with an upper-bounded steady-state error provided $\delta \in\left(0, \min \left\{\alpha^{-1}, \beta^{-1}\left(d_{\max }^{\text {out }}\right)^{-1}\right\}\right)$, specifically

$$
\lim _{k \rightarrow \infty}\left|x^{i}(k)-\frac{1}{N} \sum_{j=1}^{N} u^{j}(k)\right| \leq\left(\delta \beta \hat{\lambda}_{2}\right)^{-1} \gamma, \quad i \in\{1, \ldots, N\} .
$$

One can make similar comments to those of Remark 4.2 regarding the tuning of the performance of (30) via the design parameters $\alpha$ and $\beta$. In the following, we identify conditions, involving inputs and their differences, under which the algorithm (30) solves Problem 1 with zero steady-state error. 
Lemma 4.7 (Conditions on inputs for zero steady-state error of (30)): Let $\mathcal{G}$ be strongly connected and weight-balanced. Assume there exists $\delta \in\left(0, \min \left\{\alpha^{-1}, \beta^{-1}\left(d_{\max }^{\text {out }}\right)^{-1}\right\}\right)$ and $\alpha>0$ such that for all $i \in\{1, \ldots, N\}$, one of the following conditions are satisfied

(a) $\Delta u^{i}(k)+\delta \alpha u^{i}(k)$ converges to a common dynamics $l(k)$;

(b) $\Delta u^{i}(k+1)-\Delta u^{i}(k)+\delta \alpha \Delta u^{i}(k)$ converges to a common dynamics $l(k)$.

Then, the algorithm (30) over $\mathcal{G}$ with the given $\delta$ and $\alpha, z^{i}(0), v^{i}(0) \in \mathbb{R}$ such that $\sum_{i=1}^{N} v^{i}(0)=0$, for any $\beta>0$, makes $x^{i}(k) \rightarrow \frac{1}{N} \sum_{j=1}^{N} u^{j}(k)$, as $k \rightarrow \infty$, for all $i \in\{1, \ldots, N\}$.

\section{Dynamic average consensus with controllable rate of con- vergence and limited control authority}

In this section, we address the dynamic average consensus Problems 2 and 3. As discussed in Section 3, the goal in setting up these problems is to come up with an algorithm which is more suitable for applications where the agreement state $x^{i}$ in (3) corresponds to some physical variable such as position of a robotic system. In such networked systems, agents might have limited control authority and can not implement the high-rate commands dictated by the consensus algorithm.

Although the rate of convergence of the algorithm can be controlled by the choice of $\alpha$ and $\beta$, these variables are centralized variables and the effect is universal across the network. One can expect that a more efficient consensus algorithm is one that allows agents with limited power to move at their own pace. To this end, we make a modification to the structure of the consensus algorithm (4), 


$$
\begin{aligned}
& \dot{z}^{i}=\dot{u}^{i}-\alpha\left(z^{i}-u^{i}\right)-\beta \sum_{j=1}^{N} \mathbf{L}_{i j} z^{j}-v^{i}, \\
& \dot{v}^{i}=\alpha \beta \sum_{j=1}^{N} \mathbf{L}_{i j} z^{j}, \\
& \dot{x}^{i}=-\theta^{i}(t)\left(x^{i}-z^{i}\right)+\dot{u}^{i}-\alpha\left(z^{i}-u^{i}\right)-\beta \sum_{j=1}^{N} \mathbf{L}_{i j} z^{j}-v^{i},
\end{aligned}
$$

where $\theta^{i}:[0, \infty) \rightarrow \mathbb{R}$ is a time-varying gain which is bounded from below and above, i.e., at all $t \geq 0$ we have $0<\underline{\theta}^{i} \leq \theta^{i}(t) \leq \bar{\theta}^{i}$, for $i \in\{1, \ldots, N\}$. As we show below, agents that wish to slow down their rate of convergence use this gain to adjust it. Note the cascading structure of the algorithm. As such, the stability properties of (34a)-(34b) (information phase) are independent of (34c) and are as characterized in Section 4. The information phase allows agents to obtain the average with a convergence rate that is common across the network. The dynamics (34c) (motion phase) allows each agent $i \in\{1, \ldots, N\}$ to tweak its convergence rate by adjusting the gain $\theta^{i}$. We start our analysis by examining the rate of convergence of the algorithm (34) and establishing an upper bound on its tracking error.

Lemma 5.1 (The algorithm (34) solves Problem 2): Let $\mathcal{G}$ be strongly connected and weight-balanced. For inputs whose derivatives satisfy $\left\|\boldsymbol{\Pi}_{N} \dot{\boldsymbol{u}}\right\|_{\text {ess }}=\gamma<\infty$, for any $\alpha, \beta>0$ the algorithm (34) initialized at $x^{i}(0), v^{i}(0) \in \mathbb{R}$ such that $\sum_{i=1}^{N} v^{i}(0)=0$, then we have the same ultimate tracking error bound of (22). The rate of decay of the transient response is $\min \left\{\underline{\theta}^{i}, \alpha, \beta \hat{\lambda}_{2}\right\}$ for each agent $i \in\{1, \ldots, N\}$.

Proposition 5.1 Consider the information phase (34a)-(34b). From Theorem 4.1 and Corollary 4.1, it follows that $z^{i}-\frac{1}{N} \sum_{j=1}^{N} u^{j}(t)$ has the ultimate bound

$$
\lim _{t \rightarrow \infty} \sup \left|z^{i}(t)-\frac{1}{N} \sum_{j=1}^{N} u^{j}(t)\right| \leq\left(\beta \hat{\lambda}_{2}\right)^{-1} \gamma,
$$

and converges to this neighborhood of the input average with a rate of $\min \left\{\alpha, \beta \hat{\lambda}_{2}\right\}$. Next, consider 
the motion phase (34c), which can be written as

$$
\dot{x}^{i}=-\theta^{i}(t)\left(x^{i}-z^{i}\right)+\dot{z}^{i}, \quad i \in\{1, \ldots, N\}, \forall t \geq 0 .
$$

With the change of variables $d^{i}=x^{i}-z^{i}, i \in\{1, \ldots, N\}$, this can be equivalently written as

$$
\dot{d}^{i}=-\theta^{i}(t) d^{i}, \quad i \in\{1, \ldots, N\}, \forall t \geq 0
$$

Using the Lyapunov function $V^{i}=\frac{1}{2}\left(d^{i}\right)^{2}$, it is not difficult to show that, for $0<\underline{\theta}^{i} \leq \theta^{i}(t) \leq \bar{\theta}^{i}$, (36) is an exponentially stable system which satisfies the following bound

$$
\left|x^{i}(t)-z^{i}(t)\right|=\left|d^{i}(t)\right| \leq\left|x^{i}(0)-z^{i}(0)\right| \mathrm{e}^{-\underline{\theta}^{i} t}, \quad i \in\{1, \ldots, N\}, \forall t \geq 0 .
$$

Therefore,

$$
\left|x^{i}(t)-\frac{1}{N} \sum_{j=1}^{N} u^{j}(t)\right| \leq\left|x^{i}(0)-z^{i}(0)\right| \mathrm{e}^{-\underline{\theta}^{i} t}+\left|z^{i}(t)-\frac{1}{N} \sum_{j=1}^{N} u^{j}(t)\right|, \quad i \in\{1, \ldots, N\}, \forall t \geq 0 .
$$

Then, we conclude that (22) is satisfied. The rate of convergence of agent $i$ is $\min \left\{\underline{\theta}^{i}, \alpha, \beta \hat{\lambda}_{2}\right\}$.

As before, the design parameters $\alpha$ and $\beta$ can be used to tune the overall rate of convergence. Agents who wish to move at a slower pace can use the motion phase with $\underline{\theta}^{i} \leq \min \left\{\alpha, \beta \hat{\lambda}_{2}\right\}$ to accomplish their goal. The time-varying nature of $\theta^{i}$ allows for agents to accelerate and decelerate the convergence as desired. Notice that the ultimate error bound guaranteed by algorithm (34) is the same as the one for algorithm (4). Therefore, the local first-order filter (34c) adjusts the rate of convergence without having any adverse effect on the error bound.

Remark 5.1 (Discrete-time implementation and switching networks): The results above can be extended to switching networks and discrete-time settings. For brevity this extension is omitted. In the discrete-time implementation, it is straightforward to show that for convergence we should require $\delta \in\left(0, \min \left\{\overline{\bar{\theta}}^{-1}, \alpha^{-1}, \beta^{-1}\left(d_{\max }^{\text {out }}\right)^{-1}\right\}\right)$, where $\overline{\bar{\theta}}=\max _{i \in\{1, \ldots, N\}}\left\{\bar{\theta}^{i}\right\}$.

Next, we consider the case when saturation is present in the driving command. The following result states that, under suitable conditions, the algorithm (34) is a solution for Problem 3 with the same error bounds as if no saturation was present. 
Lemma 5.2 (The algorithm (34) solves Problem 3): Let $\mathcal{G}$ be strongly connected and weight-balanced. Suppose the driving command at each agent $i \in\{1, \ldots, N\}$ is bounded by $\bar{c}^{i}>0$, i.e., $\dot{x}^{i}=\operatorname{sat}_{\bar{c}^{i}}\left(c^{i}\right)$. Assume for every agent $i \in\{1, \ldots, N\}$, the following holds: (a) the input signal at each agent is such that $\frac{1}{N} \sum_{j=1}^{N} u^{j}$ is bounded, the input derivatives satisfy $\left\|\boldsymbol{\Pi}_{N} \dot{\boldsymbol{u}}\right\|_{\text {ess }}=\gamma<\infty$, and $\left\|\dot{u}^{i}\right\|_{\text {ess }}=\mu^{i}<\infty$; (b) $\bar{c}^{i}>\mu^{i}+\gamma$. Then, for any $\alpha, \beta>0$, and constant $\theta^{i}>0$, the algorithm (34) starting from any $x^{i}(0), v^{i}(0) \in \mathbb{R}$ such that $\sum_{i=1}^{N} v^{i}(0)=0$ satisfies that the ultimate tracking error bound (22).

Proposition 5.2 Following the proof of Lemma 5.1, for the information phase (34a) and (34b), we have (35). To complete the proof, we will show that under the given conditions for the input signals, despite the saturation, $x^{i} \rightarrow z^{i}$ asymptotically for all $i \in\{1, \ldots, N\}$. Under the saturation constraint, (34c) takes the form $\dot{x}^{i}=-\operatorname{sat}_{\bar{c}^{i}}\left(\theta^{i}\left(x^{i}-z^{i}\right)+\dot{z}\right)$, for $i \in\{1, \ldots, N\}$. The rest of the proof relays on Proposition B.3. According to this result, we need to show that a) $z^{i}$ is a bounded signal; b) $\left|\dot{z}^{i}(t)\right|<\bar{c}^{i}$ for all $t>t^{\star}$ where $t^{\star}$ is some finite time. For any given finite initial conditions and input signals with bounded average the requirement (a) is satisfied due to convergence guarantees of (34a)-(34b). In the following, we show that the requirement (b) is also satisfied due to the given assumptions. With change of variables (6b) and $\boldsymbol{y}=\boldsymbol{z}-\frac{1}{N} \sum_{j=1}^{N} u^{j} \mathbf{1}_{N}$, we can represent (34a) as $\dot{\boldsymbol{z}}=-\alpha \boldsymbol{y}-\beta \mathbf{L} \boldsymbol{y}-\boldsymbol{w}+\frac{1}{N} \sum_{j=1}^{N} \dot{u}^{j} \mathbf{1}_{N}$. Therefore,

$$
\lim _{t \rightarrow \infty}\left|\dot{z}^{i}(t)\right| \leq \lim _{t \rightarrow \infty}\left|-\alpha \boldsymbol{y}^{i}(t)-\boldsymbol{w}^{i}(t)+\frac{1}{N} \sum_{j=1}^{N} \dot{u}^{j}(t)\right|+\lim _{t \rightarrow \infty}\|\beta \mathbf{L} \boldsymbol{y}(t)\| \quad i \in\{1, \ldots, N\} .
$$

Using the results and the variables introduced in the proof of Theorem 4.1, we can show that

$$
-\alpha \boldsymbol{y}-\boldsymbol{w}+\frac{1}{N} \sum_{j=1}^{N} \dot{u}^{j} \mathbf{1}_{N}=-\left[\alpha \boldsymbol{S}_{11}+\boldsymbol{S}_{21} \quad \alpha \boldsymbol{S}_{12}+\boldsymbol{S}_{22}\right]\left[\begin{array}{c}
\boldsymbol{y}(0) \\
\boldsymbol{w}(0)
\end{array}\right]-\mathrm{e}^{-\alpha t} \boldsymbol{R} \boldsymbol{R}^{\top} \dot{\boldsymbol{u}}(0)+\dot{\boldsymbol{u}}(t),
$$

where $\boldsymbol{S}_{11}$ and $\boldsymbol{S}_{12}$ are given in (18), and we have

$$
\begin{aligned}
& \boldsymbol{S}_{21}=-\alpha \boldsymbol{R} \boldsymbol{\Phi}(t, 0)+\alpha^{2} \boldsymbol{R}\left(\int_{0}^{t} \boldsymbol{\Phi}(t, \tau) \mathrm{e}^{-\alpha \tau} d \tau\right) \boldsymbol{R}^{\top}+\alpha \boldsymbol{R} \boldsymbol{R}^{\top} \mathrm{e}^{-\alpha t} \\
& \boldsymbol{S}_{22}=\boldsymbol{r} \boldsymbol{r}^{\top}+\alpha \boldsymbol{R}\left(\int_{0}^{t} \boldsymbol{\Phi}(t, \tau) \mathrm{e}^{-\alpha \tau} d \tau\right) \boldsymbol{R}^{\top}+\boldsymbol{R} \boldsymbol{R}^{\top} \mathrm{e}^{-\alpha t}
\end{aligned}
$$


Recall that $\boldsymbol{\Phi}(t, \tau)=\mathrm{e}^{-\beta \boldsymbol{R}^{\top} \mathbf{L}(t-\tau)}$, then,

$$
\begin{aligned}
& \left\|\beta \mathbf{L} \boldsymbol{R} \int_{0}^{t} \boldsymbol{\Phi}(t, \tau) \boldsymbol{R}^{\top} \dot{\boldsymbol{u}}(\tau) d \tau\right\|=\left\|\beta \boldsymbol{R}^{\top} \mathbf{L} \boldsymbol{R} \int_{0}^{t} \mathbf{\Phi}(t, \tau) \boldsymbol{R}^{\top} \dot{\boldsymbol{u}}(\tau) d \tau\right\|= \\
& \left\|\boldsymbol{R} \mathrm{e}^{-\beta \boldsymbol{R}^{\top} \mathbf{L} \boldsymbol{R} t} \int_{0}^{t} \beta \boldsymbol{R}^{\top} \mathbf{L} \boldsymbol{R} \mathrm{e}^{\beta \boldsymbol{R}^{\top} \mathbf{L} \boldsymbol{R} \tau} \boldsymbol{R}^{\top} \boldsymbol{\Pi}_{N} \dot{\boldsymbol{u}}(\tau) d \tau\right\| \leq \\
& \left\|\boldsymbol{R} \mathrm{e}^{-\beta \boldsymbol{R}^{\top} \mathbf{L} \boldsymbol{R} t} \int_{0}^{t} \beta \boldsymbol{R}^{\top} \mathbf{L} \boldsymbol{R} \mathrm{e}^{\beta \boldsymbol{R}^{\top} \mathbf{L} \boldsymbol{R} \tau} d \tau\right\| \boldsymbol{\Pi}_{N} \dot{\boldsymbol{u}}\left\|_{\text {ess }}\right\|= \\
& \left\|\boldsymbol{R} \mathrm{e}^{-\beta \boldsymbol{R}^{\top} \mathbf{L} \boldsymbol{R} t}\left(\mathrm{e}^{\beta \boldsymbol{R}^{\top} \mathbf{L} \boldsymbol{R} t}-\boldsymbol{I}_{N}\right)\right\| \boldsymbol{\Pi}_{N} \dot{\boldsymbol{u}}\left\|_{\text {ess }}\right\| \leq\left\|\boldsymbol{\Pi}_{N} \dot{\boldsymbol{u}}\right\|_{\text {ess }}+\mathrm{e}^{-\beta \hat{\lambda}_{2} t}\left\|\boldsymbol{\Pi}_{N} \dot{\boldsymbol{u}}\right\|_{\text {ess }}
\end{aligned}
$$

Recall (21). In light of the relations above we can show that

$$
\lim _{t \rightarrow \infty}\left|\dot{z}^{i}(t)\right| \leq \mu^{i}+\gamma, \quad i \in\{1, \ldots, N\} .
$$

Therefore, there exists a finite time $t^{\star}$ such that $\left|\dot{z}^{i}(t)\right|<\bar{c}^{i}$ for all $t>t^{\star}$ and $i \in\{1, \ldots, N\}$.

\section{Dynamic average consensus with privacy preservation}

Here, we study the dynamic average consensus problem with privacy preservation. We consider adversaries that do not interfere with the implementation of the algorithm but are interested in retrieving information about the inputs, their average, or the agreement state trajectories of the individual agents. These adversaries might be internal, i.e., part of the network, or external. Internal adversaries have access at no cost to certain information that external adversaries do not. More specifically, an internal adversary has knowledge of the parameters $\alpha, \beta$ of the algorithm (4), its

corresponding row in the Laplacian matrix, and the agreement state of its out-neighbors. We also assume that the agent is aware of whether the algorithm is initialized with $\boldsymbol{v}(0)=\mathbf{0}$. We refer to the extreme case when an internal adversary knows the whole Laplacian matrix and the initial conditions of its out-neighbors as a privileged internal adversary. Regarding external adversaries, we assume they have access to the time history of all the communication messages. We refer to the extreme case when an external adversary has additionally knowledge of the parameters $\alpha, \beta$, the Laplacian matrix, and the initial conditions as a privileged external adversary. 
The next result characterizes the privacy-preservation properties of the dynamic average consensus algorithm (4) against adversaries. Specifically, we show that this algorithm satisfies Problem 4(a).

Lemma 6.1 (The algorithm (4) preserves the privacy of the local inputs against adversaries): Let $\mathcal{G}$ be strongly connected and weight-balanced. The executions of the algorithm (4) over $\mathcal{G}$ with $\alpha$, $\beta>0$, initialized at $x^{i}(0), v^{i}(0) \in \mathbb{R}$ such that $\sum_{i=1}^{N} v^{i}(0)=0$, satisfy

(a) an external (respectively internal) adversary cannot reconstruct the input of any (respectively another) agent;

(b) a privileged adversary cannot reconstruct the input of agent $i \in\{1, \ldots, N\}$ as long as there exists $\bar{t}>0$ such that $\dot{u}^{i}(t) \neq 0$ for $t \in[0, \bar{t})$.

Proposition 6.1 First, we investigate the validity of claim (a). Using the results in the proof of Theorem 4.1 and recalling the change of variables (6), the solution of the algorithm (4) for given initial conditions $x^{i}(0), v^{i}(0) \in \mathbb{R}$, for $i \in\{1, \ldots, N\}$ can be written as follows

$$
\begin{aligned}
& {\left[\begin{array}{l}
\boldsymbol{x}(t) \\
\boldsymbol{v}(t)
\end{array}\right]=\left[\begin{array}{cc}
\boldsymbol{S}_{11} & \boldsymbol{S}_{12} \\
\boldsymbol{S}_{21} & \boldsymbol{S}_{22}
\end{array}\right]\left[\begin{array}{c}
\boldsymbol{x}(0)+\left(\frac{1}{N} \sum_{j=1}^{N} u^{j}(0)\right) \mathbf{1}_{N} \\
\boldsymbol{v}(0)+\boldsymbol{\Pi}_{N}(\dot{\boldsymbol{u}}(0)+\alpha \boldsymbol{u}(0))
\end{array}\right]+\left[\begin{array}{c}
\left(\frac{1}{N} \sum_{j=1}^{N} u^{j}(t)\right) \mathbf{1}_{N} \\
\boldsymbol{\Pi}_{N}(\dot{\boldsymbol{u}}(t)+\alpha \boldsymbol{u}(t))
\end{array}\right]+} \\
& {\left[\begin{array}{c}
-\boldsymbol{R} \int_{0}^{t} \boldsymbol{\Phi}(t, \tau) \mathrm{e}^{-\alpha \tau} d \tau \boldsymbol{R}^{\top} \dot{\boldsymbol{u}}(0)+\boldsymbol{R} \int_{0}^{t} \boldsymbol{\Phi}(t, \tau) \boldsymbol{R}^{\top} \dot{\boldsymbol{u}}(\tau) d \tau \\
\alpha \boldsymbol{R} \int_{0}^{t} \boldsymbol{\Phi}(t, \tau) \mathrm{e}^{-\alpha \tau} d \tau \boldsymbol{R}^{\top} \dot{\boldsymbol{u}}(0)-\alpha \boldsymbol{R} \int_{0}^{t} \boldsymbol{\Phi}(t, \tau) \boldsymbol{R}^{\top} \dot{\boldsymbol{u}}(\tau) d \tau+\mathrm{e}^{-\alpha t} \boldsymbol{R} \boldsymbol{R}^{\top} \dot{\boldsymbol{u}}(0)-\boldsymbol{R} \boldsymbol{R}^{\top} \dot{\boldsymbol{u}}(t)
\end{array}\right],}
\end{aligned}
$$

where $\boldsymbol{S}_{11}$ and $\boldsymbol{S}_{12}$ are given in (18), and $\boldsymbol{S}_{21}$ and $\boldsymbol{S}_{22}$ are given in (37). For an external adversary that only has knowledge of the time history of $\boldsymbol{x}$, the number of unknowns in (38) (i.e., $\boldsymbol{u}(0), \boldsymbol{u}(t)$, $\dot{\boldsymbol{u}}(t), \boldsymbol{v}(t)$, for $\forall t \geq 0, \alpha, \beta$ and $\mathbf{L})$, regardless of the initial condition requirement $\sum_{i=1}^{N} v^{i}(0)=0$, is larger than the number of equations. This is true even if the inputs are static. Thus, the claim (a) for external adversaries follows. Regarding the claim (a) for internal adversaries, we consider the extreme case where the adversarial agent, say $j$, is the in-neighbor of every other agent in the network, and therefore knows the time history of the aggregated vector $\boldsymbol{x}$. Now consider (4b) for all $i \in \mathcal{V} \backslash\{j\}$. Recall that agent $j$ does not know $\mathbf{L}_{i k}, k \in \mathcal{V}$, of all agent $i \in \mathcal{V} \backslash\{j\}$. Therefore, even 
if it knows the initial condition $v^{i}(0)$, it cannot obtain $v^{i}(t), t>0$. Next consider (4a), and again assume an extreme case that the adversarial agent $j$ can numerically reconstruct $\dot{x}^{i}$ with an acceptable precision and the inputs are static. Despite these assumptions, because $u^{i}$ and $\sum_{k=1}^{N} \mathbf{L}_{i k} x^{k}, \forall t \geq 0$ of all agent $i \in \mathcal{V} \backslash\{j\}$ are unknown to agent $j$, regardless of value of $v^{i}$, this agent cannot reconstruct $u^{i}$ from (4b). This concludes validity of the claim (a) for internal agents.

Next, we examine claim (b) considering both the internal and external adversary case at the same time. For an internal adversary, assume the extreme case when it is the in-neighbor of every other agent in the network. As a result, it knows the time history of the aggregated vector $\boldsymbol{x}$. At any given $\tau>0$, using its knowledge of $\boldsymbol{x}(t)$ over $t \in[0, \tau]$ and the information on the initial conditions and the parameters of the algorithm, a privileged internal or external adversary can reconstruct $v^{i}(t)$, $i \in\{1, \ldots, N\}$, for all $t \in[0, \tau]$ by integrating (4b). The adversary can also use its knowledge of $\boldsymbol{x}(t)$ over $t \in[0, \tau]$ to construct numerically $\dot{\boldsymbol{x}}(t)$ over the same period of time. Then, the adversary using (4a), knows the right-hand side of the following equation

$$
\dot{u}^{i}+\alpha u^{i}=-\dot{x}^{i}-\alpha x^{i}-\beta \sum_{j=1}^{N} \mathbf{L}_{i j} x^{j}-v^{i}, \quad \forall i \in\{1, \ldots, N\} .
$$

Because there exists $\bar{t}>0$ such that $\dot{u}^{i}(t) \neq 0$ for $t \in[0, \bar{t})$, (39) is an ordinary differential equation (ODE) with variable $u^{i}$. The adversary does not know the initial condition $u^{i}(0)$, hence, it cannot obtain the unique solution of the ODE, i.e., the dynamic input $u^{i}$. This validates claim (b).

Remark 6.1 (Privacy preservation of static inputs against privileged adversaries): To protect local static inputs from privileged adversaries, agents can add a static or time-varying value to their inputs at the beginning for some short period of time (so that the requirement of Lemma 6.1(b) is satisfied) and then remove it. This modification does not affect the final convergence properties of the algorithm (4).

In general, the algorithm (4) does not satisfy the requirements (b) and (c) of Problem 4. Here, we 
propose a slight extension of (34) that overcomes this shortcoming. For each $i \in\{1, \ldots, N\}$, let

$$
\begin{aligned}
& \dot{z}^{i}=\dot{u}^{i}-\alpha\left(z^{i}-u^{i}\right)-\beta \sum_{j=1}^{N} \mathbf{L}_{i j} \tilde{z}^{j}-v^{i}, \\
& \dot{v}^{i}=\alpha \beta \sum_{j=1}^{N} \mathbf{L}_{i j} \tilde{z}^{j}, \\
& \dot{x}^{i}=-\theta^{i}(t)\left(x^{i}-z^{i}\right)+\dot{u}^{i}-\alpha\left(z^{i}-u^{i}\right)-\beta \sum_{j=1}^{N} \mathbf{L}_{i j} \tilde{z}^{j}-v^{i}, \\
& \tilde{z}^{i}=z^{i}+\psi(t),
\end{aligned}
$$

where $\psi:[0, \infty) \rightarrow \mathbb{R}$ is a common dynamic signal which is known to all agents. Also, $\theta^{i}:[0, \infty) \rightarrow \mathbb{R}$ such that $\underline{\theta}^{i} \leq \theta^{i}(t) \leq \bar{\theta}^{i}$ for all $t \geq 0$ is a local signal only known to agent $i$. The role of the signal $\psi$ is to conceal the final agreement value from the external adversaries to satisfy the item (b) in Problem 4. Note that, because $\sum_{j=1}^{N} \mathbf{L}_{i j}=0$, the signal $\psi$ has no effect on the algorithm execution, and therefore, the executions of algorithms (40) and (34) are the same. Consequently, Lemma 5.1 is valid for (40) as well. As agents communicate $\tilde{z}^{i}$ instead of $z^{i}$, and the signal $\psi$ is unknown to the external adversaries, recovering the steady-state solution of the algorithm is impossible for such adversaries. The agreement state equation of any agent $i$ in (40c) is a local equation, with all the components set by that agent. Therefore, $x^{i}(0)$ and $\theta^{i}$ can easily be concealed from other agents, making it impossible for adversaries to reconstruct the trajectories of $x^{i}$. This allows us to satisfy the item (c) in Problem 4. The following result shows that the algorithm (40) is privacy preserving and solves Problem 4. Its proof is a consequence of the above discussion and Lemmas 5.1 and 6.1, and is omitted for brevity.

Lemma 6.2 (The algorithm (40) solves Problem 4): Under the hypotheses of Lemma 5.1, the ultimate tracking error bound (22) is valid for all trajectories $t \mapsto x^{i}(t)$ of the algorithm (40). Furthermore,

(a) an external (respectively internal) adversary cannot reconstruct the input of any (respectively another) agent; 


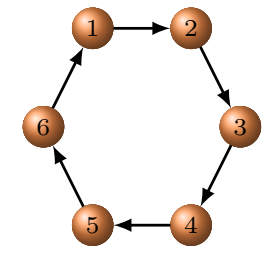

(a)

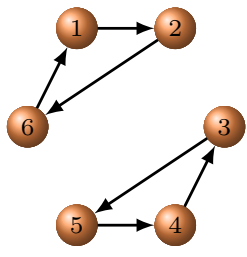

(b)

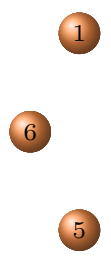

(c)

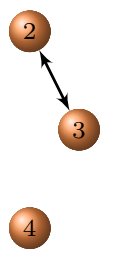

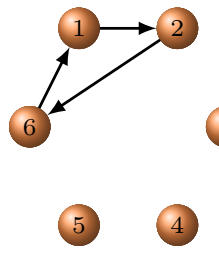

(d)

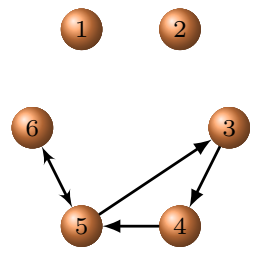

(e)

Figure 1: Weight-balanced digraphs used in simulation (all edge weights are equal to 1).

(b) a privileged adversary cannot reconstruct the input of agent $i \in\{1, \ldots, N\}$ as long as there exists $\bar{t}>0$ such that $\dot{u}^{i}(t) \neq 0$ for $t \in[0, \bar{t})$;

(c) external adversaries cannot obtain the final agreement value of the network as long as $\psi$ is unknown to them;

(d) an adversary cannot reconstruct the trajectory $t \mapsto x^{i}(t)$ of agent $i \in\{1, \ldots, N\}$ as long as $x^{i}(0)$ or $\theta^{i}$ is unknown to it.

\section{Simulations}

Here, we evaluate the performance of the proposed dynamic average consensus algorithms in a number of scenarios. Fig. 1 shows the weight-balanced digraphs employed in the simulation.

\subsection{Networks with time-varying interaction topologies}

Consider a group of 6 agents whose communication topology is time-varying. We consider the following cases for the input signals 


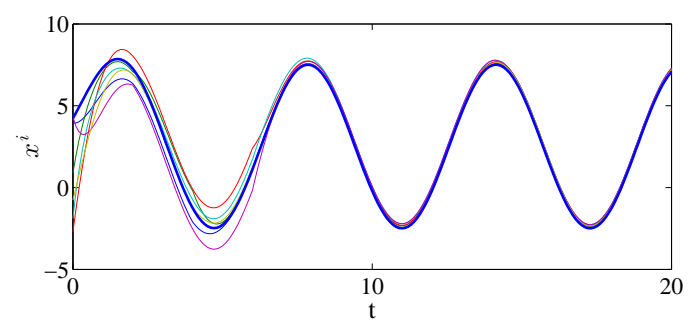

(a) Case 1

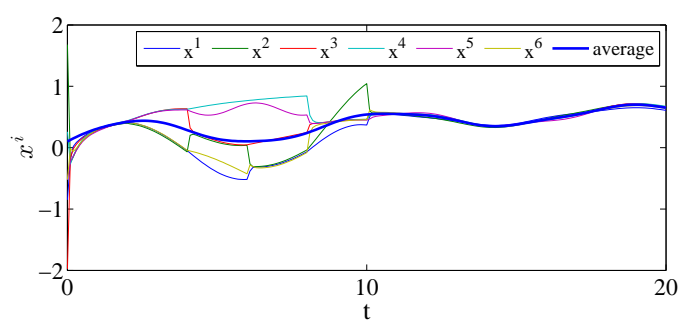

(b) Case 2

Figure 2: Simulation results for Case 1 and Case 2 of the numerical example of Section 7.1: Solid thick blue line (colored thin lines) is the input average (resp. agreement state of agents).

Case 1: $\left\{\begin{array}{l}u^{1}(t)=5 \sin t+\frac{1}{t+2}+3, \\ u^{2}(t)=5 \sin t+\frac{1}{(t+2)^{2}}+4, \\ u^{3}(t)=5 \sin t+\frac{1}{(t+2)^{3}}+5, \\ u^{4}(t)=5 \sin t+10 \mathrm{e}^{-t}+4, \\ u^{5}(t)=5 \sin t+\operatorname{atan} t-1.5, \\ u^{6}(t)=5 \sin t-\tanh t+1 .\end{array} \quad\right.$ Case $2: \quad\left\{\begin{array}{l}u^{1}(t)=0.55 \sin (0.8 t), \\ u^{2}(t)=0.5 \sin (0.7 t)+0.5 \cos (0.6 t), \\ u^{3}(t)=0.1 t \\ u^{4}(t)=\operatorname{atan}(0.5 t), \\ u^{5}(t)=0.1 \cos (2 t), \\ u^{6}(t)=0.5 \sin (0.5 t) .\end{array}\right.$

In Case 1, the communication topology iteratively changes, in alphabetical order, every two seconds among the digraphs in Fig. 1(b)-(e). In Case 2, the communication topology changes, in alphabetical order, every two seconds among the digraphs in Fig. 1(a)-(e). After $t=10$ seconds, the communication topology is fixed at the digraph in Fig. 1(a). Figure 2 shows the simulation results generated by implementing the algorithm (4) with the following parameters: in Case $1, \alpha=\beta=1$ and in Case $2, \alpha=3$ and $\beta=10$.

These examples show that, as long as the switching signal belongs to $\mathcal{S}_{\text {admis }}$, the agreement state $x^{i}$ stays bounded. In Case 1, because the input signals converge to a common function, the version of Lemma 4.3 for switching networks implies that the algorithm (4) converges to the average with zero steady-state error. However, in Case 2, we only can guarantee tracking with bounded steadystate error. During the times that the network is only weight-balanced, the error grows but still 
stays bounded. One can expect that each connected group converges to their respective input average. During these periods of time, there is no way for separate components to have knowledge of the other groups' inputs. However, once the network is strongly connected and weight-balanced, then (4) resumes its tracking of the input average across all network, as expected.

\subsection{Dynamic inputs offset by a static value}

Consider a process described by a fixed value plus a sine wave whose frequency and phase are changing randomly over time. A group of 6 agents with the communication topology shown in Fig. 1(a) monitors this process by taking synchronous samples, each according to

$$
u^{i}(m)=2+\sin (\omega(m) t(m)+\phi(m))+b^{i}, \quad m=0,1, \ldots
$$

Because of the unknown fixed bias $b^{i}$ of each agent, after each sampling, every agent wants to obtain the average of the measurements across the network before the next sampling time. Here, $\omega \sim \mathrm{N}(0,0.25), \phi \sim \mathrm{N}\left(0,(\pi / 2)^{2}\right)$, with $\mathrm{N}(.,$.$) indicating a Gaussian distribution. The data is$ sampled at 0.5 Hertz, i.e., $\Delta t=2$ seconds. The bias at each agent is $b^{1}=-0.55, b^{2}=1, b^{3}=0.6$, $b^{4}=-0.9, b^{5}=-0.6$, and $b^{6}=0.4$. Between sampling times $m$ and $m+1$, the input $u^{i}(k)$ is fixed at $u^{i}(m)$. Figure 3 shows the result of the simulation using the discrete-time consensus algorithm (30) with $\alpha=\beta=1$. The communication bandwidth is $2 \mathrm{Hertz}$, i.e., $\delta=0.5$ seconds. The application of (30) results in perfect tracking after some time as forecasted by Lemma 4.7. Notice that here as it is impossible for the agents to know $u^{i}(-1)$, the use of the algorithm in [17], which requires the agents to initialize their agreement states at $u^{i}(-1)$, results in tracking with a steady-state error.

\subsection{Limited control authority}

We use the following numerical example to demonstrate the performance of the algorithms (4) and (34) when the driving command is bounded. Consider a group of 6 agents whose communication 


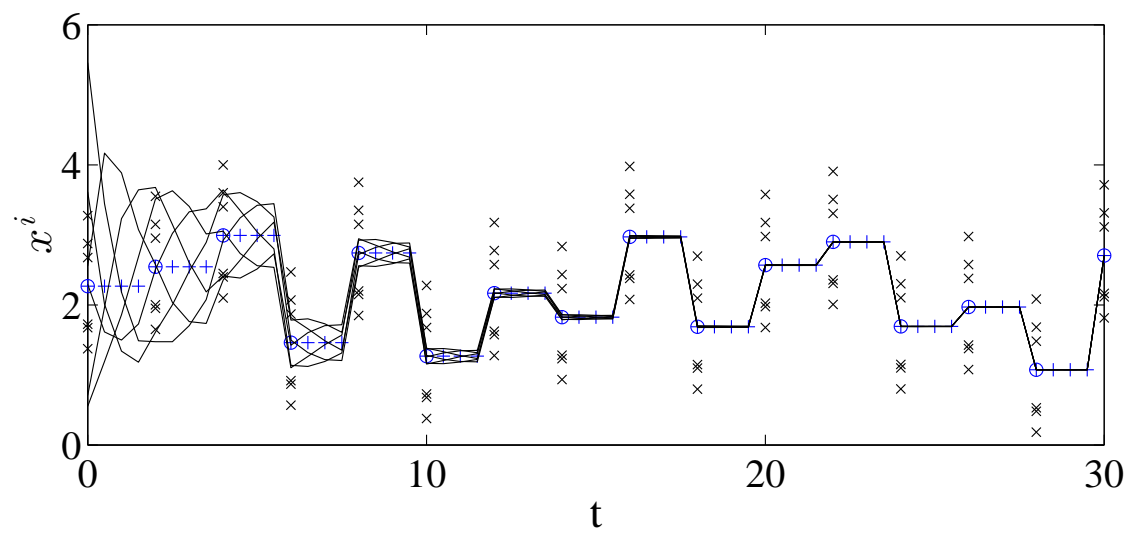

Figure 3: Simulation results for the numerical example of Section 7.2; The solid lines: the agreement states of $(30) ; \times$ : sampling points at $m \Delta t ; \circ$ : the average at $m \Delta t ;+$ : the average at $k \delta$.

topology is given in Fig. 1(a). The input signals are as follows

$$
\begin{array}{ll}
u^{1}(t)=u(t)(4 \cos (0.5 t)+10), & u^{2}(t)=u(t)(4 \tanh (t-5)+4 \tanh (t-25)+5), \\
u^{3}(t)=u(t)(4 \sin (0.5 t+1)+8), & u^{4}(t)=u(t)(4 \operatorname{atan}(0.5 t-5)-6), \\
u^{5}(t)=u(t)(\sin (2 t)-5), & u^{6}(t)=u(t)(4 \cos (0.5 t)+7),
\end{array}
$$

where $u(t)=\sum_{i=0}^{\infty}\left((-1)^{i} H(t-10 i)\right)$, in which $H$ is the step function, $H(t)=0$ if $t<0$, and $H(t)=1$ if $t \geq 0$. For both algorithms (4) and (34) we use $\alpha=10$ and $\beta=15$. In the algorithm (34) we set $\theta^{i}=1$ and we use the saturation bound $\bar{c}^{i}=15$ for all $i \in\{1, \ldots, 6\}$. Figure 4 shows the results of the simulation for these two algorithms. Using high values for $\beta$ we can reduce the tracking error, however, this results in larger driving commands. As a result, both algorithms violate the saturation bound. However, because the requirements of Lemma 5.2 are satisfied in this example, as shown in Fig. 4(b), the ultimate tracking behavior of the agreement states of the algorithm (34) despite the saturation resembles the response of the algorithm (4) in the absence of saturation bounds. There is not such guarantees for the algorithm (4) (see Fig. 4(a)). 


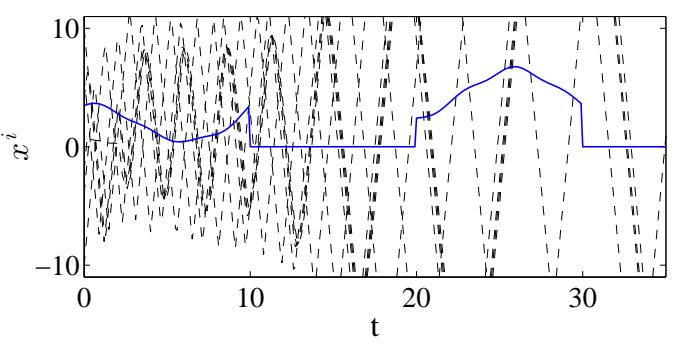

(a) Dynamic average consensus algorithm (4)

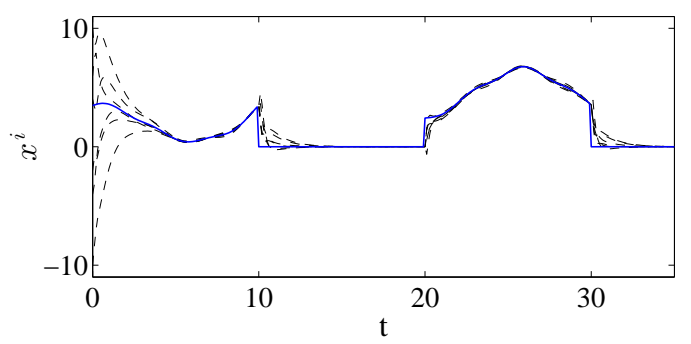

(b) Dynamic average consensus algorithm (34)

Figure 4: Simulation results for the numerical example of Section 7.3: Solid blue line (black dashed lines) is the input average (resp. agreement state of agents).

\section{Conclusions}

This paper has addressed the multi-agent dynamic average consensus problem over strongly connected and weight-balanced digraphs. We have proposed a distributed algorithm that makes individual agents track the average of the dynamic inputs across the network with a steady-state error. We have characterized how this error and the rate of convergence depend on the design parameters of the proposed algorithm, and identified special cases of inputs for which the steady-state error is zero. Our algorithm enjoys the same convergence properties in scenarios with time-varying topologies and is amenable to discrete-time implementations. We have also considered extensions of the algorithm design that can handle limited control authority and privacy preservation requirements against internal and external adversaries. Numerous avenues of research appear open for future work, including the study of discrete-time implementations with the features considered here (timevarying topologies, limited control authority, and with privacy preservation features), the design of provably-correct algorithms that do not require a priori weight-balanced interaction topologies, and the application to distributed estimation and map-merging scenarios. 


\section{References}

[1] Yang P, Freeman R, Lynch K. Multi-agent coordination by decentralized estimation and control. IEEE Transactions on Automatic Control 2008; 53(11):2480-2496.

[2] Martínez S. Distributed interpolation schemes for field estimation by mobile sensor networks. IEEE Transactions on Control Systems Technology 2010; 18(2):491-500.

[3] Cortés J. Distributed Kriged Kalman filter for spatial estimation. IEEE Transactions on Automatic Control 2009; 54(12):2816-2827.

[4] Olfati-Saber R, Shamma J. Consensus filters for sensor networks and distributed sensor fusion. IEEE Int. Conf. on Decision and Control and European Control Conference, Seville, Spain, 2005; 6698-6703.

[5] Olfati-Saber R. Distributed Kalman filtering for sensor networks. IEEE Int. Conf. on Decision and Control, New Orleans, LA, 2007; 5492-5498.

[6] Aragüés R, Cortés J, Sagüés C. Distributed consensus on robot networks for dynamically merging feature-based maps. IEEE Transactions on Robotics 2012; 28(4):840-854.

[7] Yang P, Freeman R, Lynch K. Distributed cooperative active sensing using consensus filters. IEEE Int. Conf. on Robotics and Automation, Roma, Italy, 2007; 405-410.

[8] Olfati-Saber R, Fax JA, Murray RM. Consensus and cooperation in networked multi-agent systems. Proceedings of the IEEE 2007; 95(1):215-233.

[9] Ren W, Beard RW. Distributed Consensus in Multi-vehicle Cooperative Control. Communications and Control Engineering, Springer, 2008.

[10] Ren W, Cao Y. Distributed Coordination of Multi-Agent Networks. Communications and Control Engineering, Springer: New York, 2011. 
[11] Mesbahi M, Egerstedt M. Graph Theoretic Methods in Multiagent Networks. Princeton Series in Applied Mathematics, Princeton University Press, 2010.

[12] Bullo F, Cortés J, Martínez S. Distributed Control of Robotic Networks. American Mathematical Society, Princeton University Press, 2009. Available at http://www.coordinationbook.info.

[13] Spanos D, Olfati-Saber R, Murray R. Dynamic consensus on mobile networks. IFAC World Congress, Prague, Czech Republic, 2005.

[14] Olfati-Saber R, Murray R. Consensus problems in networks of agents with switching topology and time-delays. IEEE Transactions on Automatic Control September 2004; :1520-1533.

[15] Freeman RA, Yang P, Lynch KM. Stability and convergence properties of dynamic average consensus estimators. IEEE Int. Conf. on Decision and Control, San Diego, CA, 2006; 398403.

[16] Bai H, Freeman R, Lynch K. Robust dynamic average consensus of time-varying inputs. IEEE Int. Conf. on Decision and Control, Atlanta, GA, USA, 2010; 3104-3109.

[17] Zhu M, Martínez S. Discrete-time dynamic average consensus. Automatica 2010; 46(2):322-329.

[18] Ren W. Multi-vehicle consensus with a time-varying reference state. Systems and Control Letters $2007 ; \mathbf{5 6}(2): 474-483$.

[19] Shi G, Hong Y, Johansson KH. Connectivity and set tracking of multi-agent systems guided by multiple moving leaders. IEEE Transactions on Automatic Control 2012; 57(3):663-676.

[20] Shi G, Johansson KH. Robust consensus for continuous-time multiagent dynamics. SIAM Journal on Control and Optimization 2013; 51(5):3673-3691.

[21] Hespanha J. Uniform stability of switched linear systems: extensions of lasalle's invariance principle. IEEE Transactions on Automatic Control 2004; 49(4):470-482. 
[22] Bernstein D. Matrix Mathematics: theory, facts, and formulas. 2 edn., Princeton University Press, 2009.

[23] Sontag ED, Wang Y. On characterizations of the input-to-state stability property. Systems and Control Letters 1995; 24(5):351-359.

[24] Vu L, Chatterjee D, Liberzon D. ISS of switched systems and applications to switching adaptive control. IEEE Int. Conf. on Decision and Control, Seville, Spain, 2005; 120-125.

[25] LaSalle JP. The Stability and Control of Discrete Processes, Applied Mathematical Sciences, vol. 62. Springer, 1986.

[26] Khalil HK. Nonlinear Systems. 3 edn., Prentice Hall, 2002.

[27] Sontag ED. On the input-to-state stability property. European Journal of Control 1995; 1:24-36.

\section{A Proof of the results of Section 4.3}

Here, we provide the proof of the results presented in Section 4.3.

Proposition A.1 (Proof of Lemma 4.6): We can represent the zero-system of the discrete-time algorithm (31) in the following compact form

$$
\left[\begin{array}{l}
\boldsymbol{x}(k+1) \\
\boldsymbol{v}(k+1)
\end{array}\right]=\boldsymbol{P}_{\delta}\left[\begin{array}{l}
\boldsymbol{x}(k) \\
\boldsymbol{v}(k)
\end{array}\right], \quad \boldsymbol{P}_{\delta}=\boldsymbol{I}_{2 N}+\delta \boldsymbol{A} .
$$

where $\boldsymbol{A}$ is given in (7). Then,

$$
\left[\begin{array}{c}
\boldsymbol{x}(k) \\
\boldsymbol{v}(k)
\end{array}\right]=\boldsymbol{P}_{\delta}^{k}\left[\begin{array}{l}
\boldsymbol{x}(0) \\
\boldsymbol{v}(0)
\end{array}\right] .
$$

In the proof of Lemma 4.1 we showed that the eigenvalues of $\boldsymbol{A}$ are $-\alpha$ with multiplicity of $N$ and $-\beta \lambda_{i}$ for $i \in\{1, \ldots, N\}$. Then, the eigenvalues of $\boldsymbol{P}_{\delta}$ are $1-\delta \alpha$ with multiplicity of $N$ and $1-\delta \beta \lambda_{i}$, where $i \in\{1, \ldots, N\}$. Note that the eigenvalues of $\boldsymbol{I}_{N}-\delta \beta \mathbf{L}$ are $1-\delta \beta \lambda_{i}$. Invoking [8, Lemma 
3], for a strongly connected and weight-balanced digraph, when $\delta \in\left(0, \min \left\{\alpha^{-1}, \beta^{-1}\left(d_{\max }^{\text {out }}\right)^{-1}\right\}\right)$, the eigenvalues $1-\delta \beta \lambda_{i}, i=2, \ldots, N$, are strictly inside the unit circle in the complex plane. Note that for $i=1,1-\delta \beta \lambda_{i}=1$. Therefore, we conclude that when $\delta \in\left(0, \min \left\{\alpha^{-1}, \beta^{-1}\left(d_{\max }^{\text {out }}\right)^{-1}\right\}\right)$, for a strongly connected and weight-balanced digraph $\boldsymbol{P}_{\delta}$ has an eigenvalue equal to 1 and the rest of the eigenvalues are located inside the unit circle. Therefore, $\boldsymbol{P}_{\delta}$ is a semi-convergent matrix, i.e., $\lim _{k \rightarrow \infty} \boldsymbol{P}_{\delta}^{k}$ exists. Therefore

$$
\left[\begin{array}{l}
\boldsymbol{x}(k+1) \\
\boldsymbol{v}(k+1)
\end{array}\right]-\left[\begin{array}{l}
\boldsymbol{x}(k) \\
\boldsymbol{v}(k)
\end{array}\right] \rightarrow \mathbf{0}, \quad \text { as } k \rightarrow \infty .
$$

Then,

$$
\left[\begin{array}{c}
\boldsymbol{x}(k+1) \\
\boldsymbol{v}(k+1)
\end{array}\right]-\left[\begin{array}{l}
\boldsymbol{x}(k) \\
\boldsymbol{v}(k)
\end{array}\right]=\boldsymbol{P}_{\delta}\left[\begin{array}{l}
\boldsymbol{x}(k) \\
\boldsymbol{v}(k)
\end{array}\right]-\left[\begin{array}{l}
\boldsymbol{x}(k) \\
\boldsymbol{v}(k)
\end{array}\right]=\delta \boldsymbol{A}\left[\begin{array}{l}
\boldsymbol{x}(k) \\
\boldsymbol{v}(k)
\end{array}\right] \rightarrow \mathbf{0}, \text { as } k \rightarrow \infty .
$$

As a result,

$$
\lim _{k \rightarrow \infty}\left(\left[\begin{array}{c}
\boldsymbol{x}(k) \\
\boldsymbol{v}(k)
\end{array}\right]\right)=\mu\left[\begin{array}{c}
\mathbf{1}_{N} \\
-\alpha \mathbf{1}_{N}
\end{array}\right], \quad \mu \in \mathbb{R}
$$

For a weight-balanced digraph, left multiplying the state equation of $\boldsymbol{v}$ by $\mathbf{1}^{\top}$, we obtain $\sum_{i=1}^{N} v^{i}(k+$ $1)=\sum_{i=1}^{N} v^{i}(k)$. Consequently, $\sum_{i=1}^{N} v^{i}(k)=\sum_{i=1}^{N} v^{i}(0), \forall k$. Invoking (42), then at $k=\infty$ we have $-N \mu \alpha=\sum_{i=1}^{N} v^{i}(0)$. As a result, $\mu=-\frac{\alpha^{-1}}{N} \sum_{i=1}^{N} v^{i}(0)$.

Proposition A.2 (Proof of Theorem 4.2): Consider the change of variables introduced in (6a), (33) and (9). Then (31), the equivalent representation of (30), can be expressed in the following equivalent form

$$
\begin{aligned}
& {\left[\begin{array}{l}
p_{1}(k+1) \\
q_{1}(k+1)
\end{array}\right]=\widetilde{\boldsymbol{P}}_{\delta}\left[\begin{array}{c}
p_{1}(k) \\
q_{1}(k)
\end{array}\right]} \\
& {\left[\begin{array}{c}
\boldsymbol{p}_{2: N}(k+1) \\
\boldsymbol{q}_{2: N}(k+1)
\end{array}\right]=\overline{\boldsymbol{P}}_{\delta}\left[\begin{array}{c}
\boldsymbol{p}_{2: N}(k) \\
\boldsymbol{q}_{2: N}(k)
\end{array}\right]-\left[\begin{array}{c}
\mathbf{0} \\
\boldsymbol{R}^{\top}
\end{array}\right](\Delta \boldsymbol{u}(k+1)-\Delta \boldsymbol{u}(k)+\delta \alpha \Delta \boldsymbol{u}(k)),}
\end{aligned}
$$


where $\widetilde{\boldsymbol{P}}_{\delta}=\boldsymbol{I}_{2}+\delta \widetilde{\boldsymbol{A}}$ and $\overline{\boldsymbol{P}}_{\delta}=\boldsymbol{I}_{N-2}+\delta \overline{\boldsymbol{A}}$, with $\widetilde{\boldsymbol{A}}$ and $\overline{\boldsymbol{A}}$ are defined in (10). For any given initial conditions, the solution of this difference equation is

$$
\begin{aligned}
p_{1}(k)= & p_{1}(0)-\delta \sum_{j=0}^{k-1}(1-\delta \alpha)^{j} q_{1}(0), \\
q_{1}(k)= & (1-\delta \alpha)^{k} q_{1}(0), \\
\boldsymbol{p}_{2: N}(k)= & \boldsymbol{\Phi}(k, 0) \boldsymbol{p}_{2: N}(0)-\sum_{j=0}^{k-1} \boldsymbol{\Phi}(k-1, j)(1-\delta \alpha)^{j}\left(\boldsymbol{q}_{2: N}(0)+\boldsymbol{R}^{\top} \Delta \boldsymbol{u}(0)\right)+ \\
& \sum_{j=0}^{k-1} \boldsymbol{\Phi}(k-1, j) \boldsymbol{R}^{\top} \Delta \boldsymbol{u}(j), \\
\boldsymbol{q}_{2: N}(k)= & (1-\delta \alpha)^{k}\left(\boldsymbol{q}_{2: N}(0)+\boldsymbol{R}^{\top} \Delta \boldsymbol{u}(0)\right)-\boldsymbol{R}^{\top} \Delta \boldsymbol{u}(k) .
\end{aligned}
$$

Recalling the change of variables (9), we have

$$
\boldsymbol{y}(k)=\mathbf{D}_{11} \boldsymbol{y}(0)+\mathbf{D}_{12} \boldsymbol{w}(0)-\boldsymbol{R} \sum_{j=0}^{k-1} \boldsymbol{\Phi}(k-1, j)(1-\delta \alpha)^{j} \boldsymbol{R}^{\top} \Delta \boldsymbol{u}(0)+\boldsymbol{R} \sum_{j=0}^{k-1} \boldsymbol{\Phi}(k-1, j) \boldsymbol{R}^{\top} \Delta \boldsymbol{u}(j)
$$

where

$$
\begin{aligned}
& \mathbf{D}_{11}=\left(1-\alpha \delta \sum_{j=0}^{k-1}(1-\delta \alpha)^{j}\right) \boldsymbol{r} \boldsymbol{r}^{\top}+\boldsymbol{R} \boldsymbol{\Phi}(k, 0) \boldsymbol{R}^{\top}-\alpha \boldsymbol{R}\left(\sum_{j=0}^{k-1} \boldsymbol{\Phi}(k-1, j)(1-\delta \alpha)^{j}\right) \boldsymbol{R}^{\top} \\
& \mathbf{D}_{12}=-\delta \sum_{j=0}^{k-1}(1-\delta \alpha)^{j} \boldsymbol{r} \boldsymbol{r}^{\top}-\boldsymbol{R}\left(\sum_{j=0}^{k-1} \boldsymbol{\Phi}(k-1, j)(1-\delta \alpha)^{j}\right) \boldsymbol{R}^{\top}
\end{aligned}
$$

Because $\mathbf{1}_{N}^{\top} \boldsymbol{\Pi}_{N}=\mathbf{0}$, from (33) we can deduce $\sum_{i=1}^{N} w^{i}(0)=\sum_{i=1}^{N} v^{i}(0)$. Then, it is straightforward to obtain (32).

Proposition A.3 (Proof of Corollary 4.2): We showed in Theorem 4.2 that, for any given stepsize, the bound (32) on the output $x^{i}$ of algorithm (30) holds. In the following, for the stepsizes satisfying $\delta \in\left(0, \min \left\{\alpha^{-1}, \beta^{-1}\left(d_{\max }^{\text {out }}\right)^{-1}\right\}\right)$, we find the limiting value of the terms of this bound when $k \rightarrow \infty$. Notice that $0<\delta<\alpha^{-1}$, then $0<(1-\alpha \delta)<1$. As a result, when $k \rightarrow \infty$ we have $\sum_{j=0}^{k-1}(1-\delta \alpha)^{j}=$ $(\delta \alpha)^{-1}$, leading to $\left(1-\alpha \delta \sum_{j=0}^{k-1}(1-\delta \alpha)^{j}\right) \rightarrow 0$ as $k \rightarrow \infty$. Recall $\boldsymbol{\Phi}(k, j)=\left(\boldsymbol{I}_{N-1}-\delta \beta \boldsymbol{R}^{\top} \mathbf{L} \boldsymbol{R}\right)^{k-j}$. Because $0<\delta<\beta^{-1}\left(d_{\max }^{\text {out }}\right)^{-1}$, the spectral radius of $\mathbf{\Phi}(1,0)$ is less than one, therefore $\mathbf{\Phi}(k, 0) \rightarrow 0$ and $\sum_{j=0}^{k-1} \mathbf{\Phi}(k-1, j)=\left(\delta \beta \boldsymbol{R}^{\top} \mathbf{L} \boldsymbol{R}\right)^{-1}$ as $k \rightarrow \infty$ (see [22, Fact 10.3.1.xiii]). Also, there exists 
$\omega \in(0,1)$ such that $\rho(\mathbf{\Phi}(1,0))<\omega<1$. Then $\exists \mu>0$ such that $\|\mathbf{\Phi}(k-1, j)\| \leq \mu \omega^{k-1-j}$ for $0<j \leq k-1$, [25, pp. 26]. As a result, we have

$$
\left\|\sum_{j=0}^{k-1} \mathbf{\Phi}(k-1, j)(1-\delta \alpha)^{j}\right\| \leq \mu \sum_{j=0}^{k-1} \omega^{k-1-j}(1-\delta \alpha)^{j} .
$$

Notice that

$$
\sum_{j=0}^{k-1} \omega^{k-1-j}(1-\delta \alpha)^{j}=\omega^{k-1} \sum_{j=0}^{k-1}\left(\frac{1-\delta \alpha}{\omega}\right)^{j}=(1-\delta \alpha)^{k-1} \sum_{j=0}^{k-1}\left(\frac{\omega}{1-\delta \alpha}\right)^{j} .
$$

Then, as $k \rightarrow \infty$ we have

$$
\left\|\sum_{j=0}^{k-1} \mathbf{\Phi}(k-1, j)(1-\delta \alpha)^{j}\right\| \leq \mu \sum_{j=0}^{k-1} \omega^{k-1-j}(1-\delta \alpha)^{j}= \begin{cases}\mu \omega^{k-1}\left(1-\frac{1-\delta \alpha}{\omega}\right) \rightarrow 0, & \omega>1-\delta \alpha, \\ \mu(k-1) \omega^{k-1} \rightarrow 0, & \omega=1-\delta \alpha, \\ \mu(1-\delta \alpha)^{k-1}\left(1-\frac{\omega}{1-\delta \alpha}\right) \rightarrow 0, & \omega<1-\delta \alpha .\end{cases}
$$

Invoking [22, Fact 8.18.12], we have

$$
\left\|\left(\boldsymbol{R}^{\top} \mathbf{L} \boldsymbol{R}\right)^{-1}\right\|=\sigma_{\max }\left(\left(\boldsymbol{R}^{\top} \mathbf{L} \boldsymbol{R}\right)^{-1}\right) \leq \sigma_{\max }\left(\left(\boldsymbol{R}^{\top} \operatorname{Sym}(\mathbf{L}) \boldsymbol{R}\right)^{-1}\right)=\hat{\lambda}_{2}^{-1} .
$$

Also, notice that $\forall k \geq 0$, we have $\left\|\boldsymbol{R}^{\top} \Delta \boldsymbol{u}(k)\right\|=\left\|\boldsymbol{R}^{\top} \boldsymbol{\Pi}_{N} \Delta \boldsymbol{u}(k)\right\| \leq\left\|\boldsymbol{R}^{\top}\right\|\left\|\boldsymbol{\Pi}_{N} \Delta \boldsymbol{u}(k)\right\| \leq \gamma$. Using the limiting values above, we can conclude that

$$
\left\|\sum_{j=0}^{k-1} \boldsymbol{\Phi}(k-1, j) \boldsymbol{R}^{\top} \Delta \boldsymbol{u}(j)\right\| \leq \gamma\left\|\sum_{j=0}^{k-1} \boldsymbol{\Phi}(k-1, j)\right\|=\gamma\left\|\left(\delta \beta \boldsymbol{R}^{\top} \mathbf{L} \boldsymbol{R}\right)^{-1}\right\| \leq \gamma /\left(\delta \beta \hat{\lambda}_{2}\right) .
$$

This completes the proof.

Proposition A.4 (Proof of Lemma 4.7): Using the change of variable (6a), the algorithm (30) can be stated as follows (compact form)

$$
\left[\begin{array}{c}
\boldsymbol{y}(k+1) \\
\boldsymbol{v}(k+1)
\end{array}\right]=\boldsymbol{P}_{\delta}\left[\begin{array}{l}
\boldsymbol{y}(k) \\
\boldsymbol{v}(k)
\end{array}\right]+\left[\begin{array}{c}
\boldsymbol{\Pi}_{N}(\Delta \boldsymbol{u}(k)+\alpha \delta \boldsymbol{u}(k)) \\
\mathbf{0}
\end{array}\right],
$$

where $\boldsymbol{P}_{\delta}$ is defined in (41). When condition (a) holds we have $\boldsymbol{\Pi}_{N}(\Delta \boldsymbol{u}(k)+\delta \alpha \boldsymbol{u}(k)) \rightarrow \mathbf{0}$, as $k \rightarrow \infty$. Then (43) is a linear system with a vanishing input $\boldsymbol{\Pi}_{N}(\Delta \boldsymbol{u}(k)+\delta \alpha \boldsymbol{u}(k))$. Therefore, it 
converges to the equilibrium of its zero-system. Notice that the system matrices of (43) and (31) are the same. Therefore, when $\delta \in\left(0, \min \left\{\alpha^{-1}, \beta^{-1}\left(d_{\max }^{\text {out }}\right)^{-1}\right\}\right)$, we can use result of Lemma 4.6 to conclude that $y^{i}(k) \rightarrow-\frac{\alpha^{-1}}{N} \sum_{i=1}^{N} v^{i}(0)$, for $i \in\{1, \ldots, N\}$. Because $\sum_{i=1}^{N} v^{i}(0)=0$, then we have $x^{i}(t) \rightarrow \frac{1}{N} \sum_{j=1}^{N} u^{j}(k)$ globally asymptotically for $i \in\{1, \ldots, N\}$. Next, notice that using the change of variables (6a) and (33) another equivalent representation of (30) can be stated as follows

$$
\left[\begin{array}{c}
\boldsymbol{y}(k+1) \\
\boldsymbol{w}(k+1)
\end{array}\right]=\boldsymbol{P}_{\delta}\left[\begin{array}{c}
\boldsymbol{y}(k) \\
\boldsymbol{w}(k)
\end{array}\right]-\left[\begin{array}{c}
\mathbf{0} \\
\boldsymbol{\Pi}_{N}(\Delta \boldsymbol{u}(k+1)-\Delta \boldsymbol{u}(k)+\delta \alpha \Delta \boldsymbol{u}(k))
\end{array}\right],
$$

where $\boldsymbol{P}_{\delta}$ again is defined in (41). When condition (b) holds we have $\boldsymbol{\Pi}_{N}(\Delta \boldsymbol{u}(k)+\delta \alpha \boldsymbol{u}(k)) \rightarrow$ $\mathbf{0}$ as $k \rightarrow \infty$. Then, (44) is a linear system with a vanishing input $\boldsymbol{\Pi}_{N}(\Delta \boldsymbol{u}(k+1)-\Delta \boldsymbol{u}(k)+$ $\delta \alpha \Delta \boldsymbol{u}(k))$. Then, using a similar argument used for (43) above, we can show that in (44) $y^{i}(k) \rightarrow$ $-\frac{\alpha^{-1}}{N} \sum_{i=1}^{N} w^{i}(0)$ as $k \rightarrow \infty$ for $i \in\{1, \ldots, N\}$. Using (33), we can show $\sum_{i=1}^{N} w^{i}(0)=\sum_{i=1}^{N} v^{i}(0)$. As a result $x^{i}(k) \rightarrow \frac{1}{N} \sum_{j=1}^{N} u^{j}(k)$ globally asymptotically for $i \in\{1, \ldots, N\}$.

\section{B Supporting material for the proof of Lemma 5.2}

The following results are used in the proof of Lemma 5.2.

Proposition B.1 Consider the following system where $x, w, \beta \in \mathbb{R}, \beta>0$ and $w$ is a piece-wise continuous time-varying signal

$$
\dot{y}=-\beta \operatorname{sat}_{\bar{c}}(y-w)-\beta w .
$$

Assume that $\|w\|_{\text {ess }}<\bar{c}$. Then, for any initial condition $y(0) \in \mathbb{R}, y(t) \rightarrow 0$ asymptotically.

Proposition B.2 Consider the candidate Lyapunov function $V=\frac{1}{2 \beta} y^{2}$ with derivative $\dot{V}=-y \operatorname{sat}_{\bar{c}}(y-$ $w)-y w$ along the trajectories of (45). To prove that $\dot{V}$ is negative definite, first note that because $\|w\|_{\text {ess }}<\bar{c}$, we have that if $y-w>\bar{c}$ then $y>\bar{c}+w>0$ and if $y-w<-\bar{c}$ then $y<-\bar{c}+w<0$. 
As a result,

$$
\dot{V}= \begin{cases}-y(\bar{c}+w) \leq-\left(\bar{c}-|| w \mid \|_{\text {ess }}\right)|y|<0, & \text { if } y-w>\bar{c}, \\ -y^{2}<0, & \text { if }|y-w| \leq \bar{c}, \\ -y(-\bar{c}+w) \leq-\left(\bar{c}-|| w \mid \|_{\text {ess }}\right)|y|<0, & \text { if } y-w<-\bar{c}\end{cases}
$$

All the conditions of the Lyapunov stability analysis for non-autonomous systems [26, Theorem 4.9] are satisfied globally. Therefore, $y(t) \rightarrow 0$ globally asymptotically as $t \rightarrow \infty$.

Proposition B.3 Consider the following system where $x, u \in \mathbb{R}$ and $u$ is a piece-wise continuous time-varying signal,

$$
\dot{x}=-\operatorname{sat}_{\bar{c}}(\beta(x-u)-\dot{u}),
$$

Assume $u$ and its derivative $\dot{u}$ are both essentially bounded signals, and there is some finite $t^{\star}>0$ such that for all $t \geq t^{\star},|\dot{u}(t)|<\bar{c}$. Then, for any initial condition $x(0) \in \mathbb{R}$ we have $x(t) \rightarrow u(t)$ asymptotically.

Proposition B.4 Given that (46) is ISS, c.f. [27], and since $\beta u+\dot{u}$ is bounded, for any finite initial condition $x(0)$, there is a finite $\mu(x(0))>0$ such that we have $|x|<\mu(x(0))$ for all $t \geq 0$. Under the change of variables $y=\beta(x-u)$, equation (46) can be written in the following equivalent form

$$
\dot{y}=-\beta \operatorname{sat}_{\bar{c}}(y-\dot{u})-\beta \dot{u} .
$$

Since the solutions of (46) are all bounded and because both $u$ and $x$ are bounded signals, starting from any initial condition, we have the guarantee that the solutions of (47)) are also bounded. Since the input $\dot{u}$ to the system (47) satisfies the conditions of Proposition B.1 after some finite time $t^{\star}$, we can conclude that $y(t) \rightarrow 0$, or equivalently $x(t) \rightarrow u(t)$, globally asymptotically. 